\title{
A Method of Predicting the Stresses in Adhesive Joints after Cyclic Moisture Conditioning
}

\author{
A. Mubashar ${ }^{a}$, I. A. Ashcroft ${ }^{a, *}$, G. W. Critchlow ${ }^{b}$, A. D. Crocombe \\ ${ }^{a}$ Wolfson School of Mechanical and Manufacturing Engineering, Loughborough University, \\ Loughborough, Leicestershire LE11 3TU, UK \\ ${ }^{b}$ Department of Materials, Loughborough University, Loughborough, Leicestershire LE11 3TU, UK \\ ${ }^{c}$ Division of Mechanical, Medical, and Aerospace Engineering, University of Surrey, Guildford GU2 7HX, \\ UK
}

\begin{abstract}
The durability of adhesive joints is of special concern in structural applications and moisture has been identified as one of the major factors affecting joint durability. This is especially important in applications where joints are exposed to varying environmental conditions throughout their life. This paper presents a methodology to predict the stresses in adhesive joints under cyclic moisture conditioning. The single lap joints were manufactured from aluminium alloy 2024 T3 and the FM73-BR127 adhesive-primer system. Experimental determination of the mechanical properties of the adhesive was carried out to measure the effect of moisture uptake on the strength of the adhesive. The experimental results revealed that the tensile strength of the adhesive decreased with increasing moisture content. The failure strength of the single lap joints also progressively degraded with time when conditioned at $50^{\circ} \mathrm{C}$, immersed in water, however, most of the joint strength recovered after drying the joints. A novel finite element based methodology, which incorporated moisture history effects, was adopted to determine the stresses in the single lap joints after curing, conditioning and tensile testing. A significant amount of thermal residual stress was present in the adhesive layer after curing the joints, however, hygroscopic expansion after the absorption of moisture provided some relief from the curing stresses. The finite element
\end{abstract}

\footnotetext{
* Corresponding author. Tel: +44 1509 227535; fax: +44 1509223934
}

Email address: i.a.ashcroft@lboro.ac.uk (I.A.Ashcroft) 
model used moisture history dependent mechanical properties to predict the stresses after application of tensile load on the joints. The maximum stresses were observed in the fillet areas in both the conditioned and the dried joints. Study of the stresses revealed that degradation in the strength of the adhesive was the major contributor in the strength loss of the adhesive joints and adhesive strength recovery also resulted in recovered joint strength. The presented methodology is generic in nature and may be used for various joint configurations as well as for other polymers and polymer matrix composites.

Keywords: Cyclic moisture diffusion, Epoxy adhesive, Finite element user models

\section{Introduction}

Methodologies to predict the durability of adhesive joints are of interest because of the increasing use of adhesive joints in structural applications [1-3], especially in the aerospace and automotive industries. One of the main factors affecting the durability of adhesive joints is the presence of moisture. Moisture adversely affects adhesive strength and a decrease in strength with increasing amount of moisture has been previously reported [3-5]. A number of degradation models / mechanisms have been proposed in the past, which attempt to explain the deleterious influence of water within adhesive joints [6-10].

Changes in the strength of an adhesive with moisture absorption may be determined by testing bulk adhesive samples. Tensile testing of bulk adhesive samples, conditioned at different moisture conditions, may be carried out to obtain moisture dependant stress-strain curves. For example, the moisture dependant mechanical properties of a one part rubber toughened epoxy adhesive were determined by Loh et al [11] using bulk samples of 0.4 and $0.8 \mathrm{~mm}$ thickness. The specimens were conditioned at $81.2 \%, 95.8 \%$ relative humidity $(\mathrm{RH})$ and in water at $50^{\circ} \mathrm{C}$. The stress-strain curves obtained after tensile testing showed a 
progressive decrease in the elastic modulus (E) and ultimate tensile stress ( $\left.\sigma_{\text {ult }}\right)$ with increasing moisture concentration.

When an adhesive is subjected to multiple cycles of absorption and desorption, moisture history dependence on its diffusion and mechanical properties is observed [12-15]. The moisture history dependence of a diglycidyl ether of bisphenol A (DGEBA) / dicyandiamide (DDA) epoxy polymer was investigated by Lin and Chen [13] who subjected the polymer to cycles of absorption, desorption and re-absorption at $85^{\circ} \mathrm{C}$ and $85 \% \mathrm{RH}$. The tensile strength and the elastic modulus of the polymer decreased by $29.5 \%$ and $29.1 \%$ respectively, after the first moisture absorption cycle. When the samples were dried, the tensile strength and the elastic modulus recovered to at least $90 \%$ of the unconditioned values. After the second moisture absorption cycle, the reduction in the tensile strength and the elastic modulus was 42.6\% and 54\% respectively, which was significantly greater than the reduction after the first moisture absorption cycle. Plasticisation of the adhesive was proposed as the main reason for the observed degradation in the mechanical properties of the adhesive. As plasticisation is considered potentially reversible [16], large recoveries in the elastic modulus and the tensile strength were observed after drying the sample, although, these did not return completely to the levels measured in the unconditioned state. Similar experimental observations were reported by Orman and Kerr [17] when they conditioned aluminium epoxy joints at 5\% RH and $100 \%$ RH. A decrease in the joint strength was observed with increasing exposure time. When the joints were dried after conditioning, most of the original strength was recovered. This suggested that irreversible mechanisms were also present during the moisture absorption. Moisture can affect adhesives in an irreversible manner via a number of potential mechanisms including; chemical degradation, chain scission and micro-cracking [16].

A framework, to assess the environmental degradation of adhesive joints based on moisture dependent properties has been presented by Crocombe [18], which can be implemented using 
the finite element method. The finite element approach provides a powerful tool for the application of durability prediction methods and has been used successfully in recent years $[19,20]$. Using the finite element based approach, a coupled stress-diffusion analysis was used to predict joint durability by Wahab et al [21].

In this study, single lap and butt joints were conditioned in water at $60^{\circ} \mathrm{C}$ for up to 60 weeks. A two dimensional finite element model was used to determine the moisture diffusion and swelling strains. Tensile loads were applied to the joints, which had been conditioned for various time intervals, resulting in residual swelling strains, to determine the joint stresses. A decrease in joint strength was observed with increasing moisture conditioning time and was attributed to adhesive strength degradation and increased plasticity. Similar results were reported by Broughton and Hinopoulos [22] when lap joints that had been conditioned in water were analysed using non-linear elastic-plastic finite element analysis. In most of the durability prediction studies found in the literature, the moisture concentration and stress predictions are based on the diffusion properties from a single moisture absorption. However, for joints exposed to varying moisture conditions, a predictive methodology based on moisture history dependent diffusion and mechanical properties is required to accurately predict the joint durability.

In previous work [14], a methodology for the prediction of moisture concentration in an adhesive joint after cyclic moisture diffusion was presented. This was the first step in predicting joint stresses under cyclic moisture conditions and this paper extends the cyclic moisture diffusion methodology by presenting a moisture history dependent stress prediction methodology for adhesive joints subjected to variable moisture conditions. Coupling between the cyclic moisture diffusion prediction and stress prediction methods was established by means of moisture and moisture history dependent material properties. The moisture dependant mechanical properties of the adhesive were determined by tensile testing bulk 
adhesive samples conditioned for multiple diffusion cycles. The proposed methodology was used to study the stresses in single lap joints, conditioned for different time intervals at $50^{\circ} \mathrm{C}$ in water. Stresses due to thermal, hygroscopic and mechanical loadings were included in the analysis in order to observe their effects on joint strength.

\section{Material Characterisation}

The structural adhesive FM73-M, from Cytec Engineered Materials Ltd, New Jersey, USA, was used to prepare bulk adhesive samples and aluminium alloy 2024 (Al2024), together with the FM73-M and BR127 primer were used to manufacture single lap joints. FM73-M is a single part, heat setting, rubber toughened epoxy, which has a manufacturer's recommended curing temperature of $120^{\circ} \mathrm{C}$. FM73-M was chosen based on its high strength, good resistance to moisture, broad applicable temperature range and wide use in the aerospace industry. The adhesive comes in film form and has a polyester knit carrier for support and handling. This also ensures good uniformity in layer thickness when applied in a bonded joint. BR127 is an epoxy-phenolic primer. Al2024 is a good choice for high strength to weight ratio structures such as those required in the aerospace industry where it has been used in wing and fuselage construction for many years. It has excellent machinability and fair workability and corrosion resistance.

The cyclic moisture diffusion properties of the adhesive have been reported in [14]. The moisture dependant mechanical properties of the adhesive were determined by tensile testing bulk adhesive samples, which were cut from cured sheets of the bulk adhesive according to sample size 5B of BS EN ISO 527-2:1996 [23]. The tensile test samples were conditioned at $50^{\circ} \mathrm{C}$, immersed in water, for absorption, desorption and re-absorption cycles. Experimental evidence has shown that moisture absorption under $100 \% \mathrm{RH}$ and water immersion environmental conditions is generally similar [24, 25]. At preselected time intervals, samples 
were withdrawn from the conditioning environment and tensile testing was carried out using an Instron 3366 dual column testing machine manufactured by Instron, Worwood, MA, USA, with a displacement rate of $1 \mathrm{~mm} / \mathrm{min}$. At least three tensile samples were tested at each selected time interval. Strain was measured using a strain gauge based contact extensometer. The guage length of the extensometer was $10 \mathrm{~mm}$ and knife edges of the extensometer were in contact with the bulk specimen. The specimens were wrapped with cling film to minimise any changes in moisture concentration during the transportation to the test machine and tensile testing. Single lap joints were used to determine changes in joint strength after moisture diffusion. The adherends of the single lap joints were manufactured from unclad Al2024, which was heat treated to the T3 state [26]. Its mechanical and thermal properties are given in Table 1.

The joints were prepared according to BS ISO 4587:2003 [27] and their configuration and geometry are shown in Figure 1. The adherends were cut from 3.2 mm thick Al2024 T3 sheet and the surface was prepared by degreasing followed by ACDC anodising [28]. ACDC anodising is an environmental friendly process, which is free from the hexavalent chromium found in the aerospace industry standard chromic acid anodising (CAA). Similar durability performance was observed while tensile testing of adhesive joints prepared using ACDC anodising and CAA.

During ACDC anodising, the adherends act as anodes in the DC phase and alternatively as anodes and cathodes in the AC phase. The adherends were suspended in an aqueous solution of $2.5 \%$ phosphoric plus $2.5 \%$ sulphuric acid. An alternating current (AC) was applied at a voltage of $15 \mathrm{~V}$ for 2 minutes at $35^{\circ} \mathrm{C}$. This process was followed by the application of direct current (DC) with a voltage of $20 \mathrm{~V}$ for 10 minutes at $35^{\circ} \mathrm{C}$. The adherends were then rinsed with water and dried in air. The ACDC anodising process provides a high energy, porous oxide surface on the adherends, which is ideal for adhesive bonding, with a dense, corrosion 
resistant layer adjacent to the aluminium. Further details of the ACDC pretreatment may be found in [28].

The ACDC pretreatment was followed by the application of BR127 corrosion inhibiting primer. After application, the primer was air dried for 30 minutes and then cured for one hour at $120^{\circ} \mathrm{C}$. The adhesive was brought to room temperature from its storage temperature of $-24^{\circ} \mathrm{C}$ before bonding. During curing, the adhesive and the adherends were held together by clips. The bondline thickness was maintained by the carrier in the adhesive film. The adhesive was cured at $120^{\circ} \mathrm{C}$ for one hour, resulting in a bondline thickness of $0.12 \pm 0.02$ mm. Fillets were formed at the ends of the overlap due to out-flow of the adhesive and the average size of the fillets was $1 \mathrm{~mm}$.

A set of three joints was tensile tested at room temperature using a Hounsfield H20K-W tensometer (Tinius Olsen Ltd., Philadelphia, USA) to obtain the failure strength of the unconditioned joints. The rest of the joints were conditioned in water at $50^{\circ} \mathrm{C}$. Sets of six joints were removed from the conditioning environment at predefined intervals of $7,14,28$, 56 and 182 days. Three joints from the extracted set were immediately tensile tested and the remaining three joints were placed in an oven for drying at $50^{\circ} \mathrm{C}$. The drying time for the joints was the same as the absorption time for each joint. After drying, the joints were brought to room temperature in a desiccator and tensile testing was carried out. The joints dried after conditioning will hereafter be referred to as "dried joints". Further details of the results from the single lap joint tests can be found in [29].

\section{Experimental Results}

Stress as a function of strain at different moisture conditions for the bulk adhesive tensile specimens is plotted in Figure 2. The plots are an average of results obtained after testing 
three specimens at each moisture condition and the stresses had a standard deviation of \pm 2.6 $\mathrm{MPa}$. The moisture content in each bulk adhesive specimen is given as the ratio of moisture content by weight at any time, $M_{t}$, measured by gravimetric means, to the saturated moisture content, $M_{\infty}$, i.e. $M_{t} / M_{\infty} . M_{\infty}$ was determined using a dual Fickian model [11] for absorption and a Fickian diffusion model for the desorption. During the first absorption cycle, an increase in strain to failure, over unconditioned specimens, was observed for all moisture concentrations. The increased strain to failure may be due to plasticisation of the adhesive by the absorbed moisture. In dried bulk adhesive samples, the strain to failure decreased to a value less than the unconditioned samples, which represents a brittle failure compared to the unconditioned and conditioned specimens and is further indicative of irreversible change in the adhesive after moisture conditioning. An increase in strain to failure occurred after the second moisture absorption cycle, which, again, is consistent with the plasticisation effects of moisture.

The effect of moisture cycling on the elastic modulus and ultimate tensile stress, as determined from the stress-strain curves, can be seen in Figure 3. The lines joining the experimental data points are to show trends only and join the average values at each data point. A progressive decrease in elastic modulus with increasing moisture concentration was observed during the first absorption cycle. At relatively high moisture concentrations, above $2 \mathrm{wt} \%$, the change in elastic modulus became minimal. A similar trend was observed in ultimate tensile stress, where an increase in moisture content decreased the ultimate tensile stress. There was again a progressive decrease observed from the unconditioned state to samples with 2 wt\% moisture, however, no change in ultimate tensile stress was observed above 2 wt\% moisture. The minimal change in elastic modulus and ultimate tensile stress above $2 \mathrm{wt} \%$ moisture indicates that most of the degradation in the mechanical properties took place earlier in the absorption cycle. The elastic modulus and ultimate tensile stress for 
dried bulk adhesive samples regained $95 \%$ and $93 \%$ of their unconditioned values, respectively. This shows that a large portion of the degradation of the adhesive was reversible and may be attributed to plasticisation of the adhesive, which is considered a reversible mechanism [16]. However, some irrecoverable damage occurred in the adhesive, which may be proposed to occur by chemical interaction of moisture and the adhesive, micro cracking of the adhesive or the leeching of material. In this research, leeching of the bulk adhesive samples was investigated by comparing the IR-Spectroscopy results of the water used for conditioning the bulk adhesive sample with deionised water. No significant change in chemical composition of the water was observed at the end of the conditioning period. Following a second absorption cycle with a $3.7 \mathrm{wt} \%$ moisture uptake, elastic modulus and ultimate tensile stress decreased more than in the first absorption cycle. This may be as a consequence of the irreversible damage present in the adhesive after the first absorption.

The change in failure load of the single lap joints with conditioning time is given in Figure 4. The joints showed a decrease in the failure load with increasing conditioning time. It has been proposed that moisture changes the strength of a joint through a change in the adhesive properties and potentially also by attacking the adhesive-adherend interface [3]. However, utilising advanced surface preparation methods, such as the ACDC anodisation process used in the present research, the effect of water at the interface is minimised, resulting in durable structures. This is confirmed by the large strength recovery, at least $98 \%$ of unconditioned strength, observed in the dried joints. As a result, it is proposed that most of the joint strength degradation was due to the plasticisation of the adhesive layer. The failure surface of a single lap joint failed in tension after conditioning for 182 days is shown in Figure 5.

The two main types of failure observed in the joints were cohesive failure and apparent interfacial failure. Cohesive failure in the adhesive layer was observed in the single lap joints 
tested in the unconditioned state. In the conditioned joints, cohesive failure predominated in joints tested after 7, 14, 28 and 56 days, whereas, a mixed failure was observed in the joints conditioned for 182 days. However, patches of apparent interfacial failure were present to some degree on most of the failure surfaces. A similar pattern of cohesive or mixed failure was observed in the dried joints.

\section{Stress Prediction under Cyclic Moisture Conditions}

A cyclic moisture diffusion prediction methodology was presented in [14], which is able to predict the moisture concentration over multiple cycles of absorption and desorption. The methodology was implemented by using a user subroutine in the commercially available finite element code Abaqus developed by Dassault Systemes, Providence, RI, USA. The moisture history of the adhesive was maintained by use of scalar internal state variables. In this paper, the cyclic moisture diffusion prediction methodology is coupled with a cyclic stress prediction method for the prediction of joint stresses when subjected to variable environmental conditions. The predicted stress or strains may be used with a suitable failure criterion to predict the strength of adhesive joints. The methodology will be referred to as the variable moisture and stress prediction methodology in the rest of the paper.

The overall framework for stress prediction under cyclic moisture conditions is given in Figure 6. The moisture history dependence of mechanical properties was introduced in the model by using field variables. The elastic and plastic properties of the adhesive were considered functions of; (i) the normalised moisture concentration, (ii) the moisture history, in the form of the number of diffusion cycles, and (iii) the diffusion process, i.e. either absorption or desorption. This dependency is illustrated by Equations (1) and (2).

$$
E\left(C_{t}, F V 1, F V 2\right)
$$




$$
\varepsilon^{p l}\left(C_{t}, F V 1, F V 2\right)
$$

where $\varepsilon^{p l}$ is the plastic strain of the adhesive, $C_{t}$ is the normalised moisture concentration at time $t$ and $F V 1$ and $F V 2$ are two field variables. FV1 represents the moisture history in the form of the number of absorption and desorption cycles and $F V 2$ represents the nature of the diffusion process i.e. absorption or desorption. The moisture history and moisture process are stored in state variables in the form of a spatially resolved field for the adhesive layer. The Abaqus user subroutine USDFLD was used to define coupling between the field variables and the state variables. USDFLD allows the definition of field variables at a material point as a function of time or any available material point quantity [30]. The moisture history, normalised concentration and moisture process definitions required for the predictive stress model were obtained from a cyclic moisture diffusion analysis.

\section{Comparison of Cyclic Stress Predictions with Experimental Results}

To verify the cyclic stress prediction methodology, a tensile specimen of the adhesive was modelled to predict the stress after cyclic moisture diffusion and the results were compared with the experimental data. The geometry of the tensile specimen, along with the finite element model is shown in Figure 7. A three dimensional model of the sample gauge length was used for the analysis. Symmetry enabled this to be represented by a quarter of the geometry, as shown in Figure 7b. The three dimensional model was necessary for the correct application of moisture boundary conditions. The model was meshed with eight node, continuum brick elements, which provide good response under large displacements [31]. In dog bone tensile samples of ductile material, failure generally occurs in the form of a cupcone fracture after necking of the specimen. Necking starts to develop at voids or imperfections present in the material. Such imperfections or voids are not present in the finite 
element model where a perfect material is assumed. In order to closely represent the experimentally observed deformation and necking of the tensile samples, a small geometric imperfection was introduced by moving the nodes along line $\mathrm{Y}-\mathrm{Y}$ towards line Z-Z by 0.01 mm, which is very small, compared to the overall dimensions of the specimen. A biased mesh with higher number of elements was used in the middle of the specimen.

The stress-strain response of the model was determined for four different moisture conditions; (i) unconditioned, (ii) first absorption for 58 days, (iii) dried after first absorption and (iv) second absorption for 83 days. The moisture conditions were selected based on the available experimental data. Moisture concentration in the tensile specimen, at selected moisture conditions of 58 and 83 days, was determined by diffusion analysis using the cyclic moisture diffusion methodology detailed in [14]. A dual Fickian model [11] was used for the prediction of moisture concentrations after both the first and the second absorption whilst a Fickian diffusion model was used for the desorption. The normalised moisture concentrations, at the middle of the tensile specimen, are plotted in Figure 8.

The predicted concentration and moisture history were used as initial conditions in the stress analysis of the tensile specimen. Moisture history dependant elastic-plastic properties were used for the adhesive, based on the experimental stress-strain curves given in Figure 2. The yield surface of the adhesive has hydrostatic stress dependence and a linear Drucker Prager model was used to define the yield surface where the yield function, $F$, is given by [32]:

$$
F=t-p \tan (\beta)-d=0
$$

where

$$
t=\frac{q}{2}\left[1+\frac{1}{K}-\left(1-\frac{1}{K}\right)\left(\frac{r}{q}\right)^{3}\right]
$$




$$
\begin{gathered}
q=\sqrt{3 J_{2}} \\
d=\left(1-\frac{1}{3} \tan (\beta)\right) \sigma
\end{gathered}
$$

where $\beta$ is the material angle of friction and $K$ is the flow stress ratio, $q$ is von Mises equivalent stress, $r$ is the third stress invariant, $p$ is hydrostatic stress, $J_{2}$ is the second stress invariant and $\sigma$ is the uniaxial compressive yield stress. The Drucker Prager model constants were $\beta=27.8^{\circ}$ and $K=0.85$ [32]. Comparisons of the stress-strain curves obtained from the finite element model and the experimental tensile tests are shown in Figure 9. Excellent correlation between the predicted and experimental results can be seen for all the selected moisture conditions. Strain to failure from the finite element analysis and the experimental values were in good agreement.

\section{Single Lap Joints under Cyclic Moisture Conditions}

The cyclic stress prediction methodology was then used to predict the stresses in single lap joints, which were conditioned using moisture absorption and desorption cycles, as discussed in Section 2. Geometric and loading symmetry allowed the modelling of one half of the single lap joint, as shown in Figure 10a. Fillets were included at the end of the adhesive layer based on the average dimensions measured from manufactured joints. A three dimensional model, meshed with linear eight node, continuum brick elements, was used for the analysis. A mesh sensitivity study was carried out to select the element size. Since the stresses in the adhesive layer were of interest, the element size in the adhesive layer was doubled in each subsequent model and stresses were compared to select the mesh size. The maximum mesh size where stress convergence was achieved was selected. A minimum element size of $0.12 \mathrm{x}$ $0.04 \times 0.12 \mathrm{~mm}$ was used in the adhesive layer, as shown in Figure 10b. Three elements were 
used across the adhesive thickness. A continuous mesh was used to mesh the adherends and the adhesive, thus extra constraints were not required to join the adhesive with the adherends. During the joint manufacturing, conditioning and testing; thermal, hygroscopic and mechanical stresses were generated in the single lap joints and a multi-step analysis was carried out to determine the stresses after different processing stages. Each processing step was modelled using a corresponding numerical procedure, as shown in Figure 11.

In the first step, a thermo-mechanical analysis was used to determine the thermal residual stresses in the adhesive and adherends after curing. Thermal properties for the Al2024 T3 adherend material and the FM73 adhesive are given in Table 1 and Table 2, respectively. The analysis started by heating the joint to $120^{\circ} \mathrm{C}$ from room temperature of $20^{\circ} \mathrm{C}$, as occurs at the start of the curing process. At the curing temperature, the adhesive was considered stress free and cooling of the joint to the conditioning temperature of $50^{\circ} \mathrm{C}$ was carried out, which resulted in thermal stresses in the joint.

In the second step, the thermal stresses were used as an initial condition and a hygromechanical analysis was carried out to determine the moisture concentrations and hygroscopic stresses in the joint. As with the bulk adhesive specimens, the moisture concentrations in the adhesive layer were determined using the cyclic moisture diffusion methodology detailed in [14] with a dual Fickian model for moisture absorption and a Fickian model for moisture desorption. The moisture boundary conditions were applied in the form of normalised moisture concentrations and region of application of moisture boundary condition is shown in Figure 10a. Diffusion analysis was carried out for durations of 7, 14, 28, 56 and 182 days. Each absorption analysis was followed by a desorption analysis of the same duration to determine the moisture distribution after drying. As the adherends were prepared by degreasing and the ACDC pretreatment, which promoted good wetting of the adherends, 
the potential of enhanced moisture diffusion along the interface was reduced and, hence the only moisture path considered in this case was through the adhesive. However, it should be noted that interfacial diffusion may be readily included in the proposed methodology by using a faster diffusion rate in the interfacial layer, if required.

In the final step, a structural analysis was performed and a load of $6 \mathrm{kN}$ was applied to determine the stresses under tensile loading. The moisture history, normalised moisture concentration and stresses from the hygroscopic analysis were used as initial conditions for the stress prediction. A load of $6 \mathrm{kN}$ was selected as it produced plastic deformation in the

unconditioned joints during finite element analysis. Since a strength of materials based method is used to compare stresses in the joints conditioned for different moisture levels, a load producing plastic deformation in joints was selected for comparison of stresses. The load of $6 \mathrm{kN}$ produced plasticity in the unconditioned adhesive joints while the conditioned adhesive joints exhibited plastic deformation at different loads, lower than $6 \mathrm{kN}$, depending upon the amount of moisture in the adhesive.

\section{Stresses in Single Lap Joints}

Stresses in the single lap joints developed during manufacturing, conditioning and testing. Before discussing the development of stresses in the joints, the moisture concentration in the joints is considered, which is the source of hygroscopic stresses. Figure 12 plots the normalised moisture concentration in the adhesive layer, along line A-A as shown in Figure 10a, after different periods of moisture absorption. The moisture concentration in the adhesive layer increases with conditioning time; however, saturation is not achieved, even after 182 days of exposure. The shape of the moisture absorption curves is notably different from a typical Fickian diffusion curve owing to the use of the dual Fickian model. Figure 13 shows the normalised moisture concentration after the desorption of moisture. All samples 
were dried for the same duration as they were conditioned. Although the desorption rate was higher than the absorption rate [14], moisture was still present in the adhesive layer for all desorption time periods. Since the adhesive layer had low moisture concentration in the middle of the overlap after absorption, the desorption boundary condition caused the moisture to desorb from exposed surfaces of the adhesive layer while moisture absorption continued towards the inner regions of the adhesive. Thus two simultaneous processes of absorption and desorption occurred in the adhesive layer with pockets of high moisture concentration between the overlap end and middle being observed at the end of the desorption cycle. The moisture in the adhesive layer after desorption is referred to as the residual moisture. The joints dried for 7, 14 and 28 days showed peaks of the residual moisture between the end and the middle of the overlap region, that can be seen as localised maxima in the normalised concentrations in Figure 13. In contrast, the maximum amount of residual moisture was seen in the middle of the overlap in joints dried for 182 days. The amount of residual moisture is relatively high but it should be noted that this residual moisture is after 182 days of desorption and the joint will continue to desorb moisture if the desorption is continued.

The development of stresses, during manufacturing, conditioning and testing, in a single lap joint conditioned for 7 days is shown in Figure 14. The stresses were plotted along line A-A shown in Figure 10a. The dashed vertical lines represent the ends of the adherend overlap. The mismatch of thermal expansion coefficients of the adhesive and the adherends caused significant thermal longitudinal stresses in the adhesive layer. Both the longitudinal and the peel thermal stresses are fairly uniform over the central region of the overlap, varying at the ends. The presence of highest stresses in the overlap end and fillet regions indicate that these are potential areas for crack initiation. 
When the joints were exposed to moisture, the diffusing moisture caused plasticisation and swelling of the adhesive. After conditioning for 7 days, the moisture concentration had significantly increased in the fillets and areas close to the overlap ends and a drop in residual longitudinal stress was observed in these areas. The residual peel stress close to the fillet edges also decreased with moisture ingress and the maximum peel stress moved from the edges of the fillet to inside the fillet. This may be attributed to the relief of the thermal stress by hygroscopic swelling of the adhesive, which shrank earlier due to cooling from the curing temperature. Application of a tensile load of $6 \mathrm{kN}$ resulted in high longitudinal and peel stresses in the adhesive layer. The highest peel stresses after conditioning and tensile load application were at the overlap ends. When the tensile load was applied after drying of the joints, the highest peel stress was in the same region as it was in the conditioned joints, which indicates that failure in both the conditioned and dried joints will be initiated close to the overlap end.

The development of longitudinal and peel stresses for joints conditioned for 182 days is plotted in Figure 15. The thermal residual stresses were the same for all the joints as they were cured under same conditions. The moisture content increased through the overlap length after 182 days conditioning and the highest peel stress moved from the fillet edge to the end of the overlap. The shift in highest peel stresses may be attributed to the relief of thermal stress by hygroscopic expansion. When a tensile load of $6 \mathrm{kN}$ was applied to the joints, the highest longitudinal and peel stress remained at the overlap ends. This suggests that failure in 182 days conditioned joints will also initiate close to the overlap end. Although the peak peel stresses in Figure 14 and Figure 15 are of similar magnitude, the experimental failure load of the joints has been shown to decrease with increasing moisture conditioning time, as shown in Figure 4. Thus the same level of stresses at loads of $6 \mathrm{kN}$ conditioned for 7 and 182 days show that the 182 days conditioned joints will fail earlier owing to the less overall strength. 
Also the crack propagation through the joint may be different because of the plasticisation of the adhesive by water.

As discussed above, it can be seen that moisture absorption provides some relief from thermal residual stresses, whilst the highest peel stress moves from the fillet edges to the overlap end with increasing amount of moisture in the adhesive layer. The joints conditioned for 14, 28 and 56 days showed stress development patterns with similar trends. It may be noted from the stresses developed in conditioned and dried joints that the highest stresses exist in fillet or overlap end regions.

\section{Discussion}

A progressive decrease in the strength of the single lap joints was observed after conditioning in water for different time intervals. The decrease in the joint strength may be owing to the degradation of the adhesive and/or the adherend-adhesive interface. However, as mentioned earlier, interfacial moisture effects are minimised by using adherends with high quality surface treatments such as anodising. Anodising also provides a highly porous oxide structure for the penetration of the primer and thus mechanical interlocking would occur at the interface. This makes a complete replacement of the interface with water unlikely. When the joints were dried after conditioning, they recovered at least $90 \%$ of their unconditioned strength. The large recovery in the joint strength may be attributed to the recovery of the strength of the bulk adhesive. A similar amount of recovery in the bulk adhesive strength was also observed i.e. 92\% of the unconditioned strength. This also suggests that the major reduction in the joint strength was because of the effect of moisture on the bulk adhesive properties. 
The thermal and hygrothermal stresses did not produce any plastic deformation in the adhesive layer. However, plastic deformation was predicted by finite element model after the application of a $6 \mathrm{kN}$ tensile load, as shown in Figure 16. The plastic deformation starts in the centre of the adhesive layer and expands across the width of the joint, in the $\mathrm{Z}$ direction. As the load increases during tensile testing, the plastic deformation zone increases in size and grows towards the middle of the overlap i.e. in the $\mathrm{X}$ direction. The plastic zone is larger at the centre of the adhesive layer than at the edges.

The adhesive plastic deformation development in joints, conditioned at $50^{\circ} \mathrm{C}$, is similar to the unconditioned joints. The maximum plastic strain was greater in the joints conditioned for 182 days than in those conditioned for 7 days. This is because of the degradation of adhesive stiffness with increasing moisture absorption. Residual moisture was present in the joints dried after conditioning, which affected the plastic deformation of the adhesive layer and thus the adhesive strength under tensile loading. In joints conditioned for 182 days and dried, a larger plastic deformation zone is present and the maximum plastic strain is lower than in the unconditioned joints. This decrease in the maximum plastic strain may be attributed to the spread of plastic deformation over a larger area in the dried joints.

\section{Conclusions}

A variable moisture and stress prediction methodology is presented that is capable of predicting the moisture and stresses in adhesive joints under varying moisture conditions. A finite element analysis of single lap joints based on the proposed methodology predicted that the residual curing stresses in single lap joints are of significant magnitude and hygroscopic stresses provide some relief of curing stress. The magnitude of the peel stresses after the tensile loading was similar at all moisture conditions, which suggested that the major reduction in the joint strength occurred owing to the strength reduction in the adhesive 
strength. The dried joints also showed similar peel stresses. The single lap joints showed a large amount of strength recovery after drying and it may be attributed to the mainly reversible degradation of the adhesive and interphasial polymer. The generic nature of the proposed methodology makes it applicable to most types of adhesive joint configurations. By changing the diffusion properties of the material, the variable moisture diffusion prediction methodology may not only be used to predict the moisture diffusion in different types of adhesive but also in polymers and polymer matrix composites. The use of appropriate moisture dependent mechanical properties allow the use of joint adherends made up of either metals or composites and a suitable failure criterion may be used for determining the joint residual strength. 


\section{References}

[1] Grant, L.D.R., Adams, R.D., and da Silva, L.F.M., International Journal of Adhesion and Adhesives. 29, 405-413 (2009).

[2] da Silva, L.F.M., das Neves, P.J.C., Adams, R.D., and Spelt, J.K., Int. J. Adhesion and Adhesives. 29, 319-330 (2009).

[3] Minford, J.D., Handbook of Aluminum Bonding Technology and Data, (Marcel Dekker, Inc., New York, 1993).

[4] Hand, H.M., Arah, C.O., McNamara, D.K., and Mecklenburg, M.F., Int. J. Adhesion and Adhesives. 11, 15-23 (1991).

[5] Liljedahl, C.D.M., Crocombe, A.D., Wahab, M.A., and Ashcroft, I.A., The Journal of Adhesion. 82, 1061-1089 (2006).

[6] Bowditch, M.R., Int. J. Adhesion and Adhesives. 16, 73-79 (1996).

[7] Apicella, A., Nicolais, L., Astarita, G., and Drioli, E., Polymer. 20, 1143-1148 (1979).

[8] De'Nève, B. and Shanahan, M.E.R., Polymer. 34, 5099-5105 (1993).

[9] Katya, I.I., Richard, A.P., and Affrossman, S., J. Appl Polym Sci. 84, 1011-1024 (2002).

[10] Liu, J., Lai, Z., Kristiansen, H., and Khoo, C. Proceedings of 3rd International Conference on Adhesive Joining and Coating Technology in Electronics Manufacturing (1998).

[11] Loh, W.K., Crocombe, A.D., Wahab, M.A., and Ashcroft, I.A., Int. J. Adhesion and Adhesives. 25, 1-12 (2005).

[12] Brewis, D.M., Comyn, J., and Tegg, J.L., Polymer. 21, 134-138 (1980).

[13] Lin, Y.C. and Chen, X., Polymer. 46, 11994-12003 (2005).

[14] Mubashar, A., Ashcroft, I.A., Critchlow, G.W., and Crocombe, A.D., J. Adhesion. 85, 711-735 (2009).

[15] Hua, Y., Crocombe, A.D., Wahab, M.A., and Ashcroft, I.A., International Journal of Adhesion and Adhesives. 28, 302-313 (2008).

[16] Shuangyan Xu, Dillard, D.A., and Dillard, J.G., Int. J. Adhesion and Adhesives. 23, 235-250 (2003).

[17] Berens, A.R. and Hopfenberg, H.B., Polymer. 19, 489-496 (1978).

[18] Crocombe, A.D., Int. J. Adhesion and Adhesives. 17, 229-238 (1997). 
[19] Hua, Y., Crocombe, A.D., Wahab, M.A., and Ashcroft, I.A., The Journal of Adhesion. 82, 135-160 (2006).

[20] Liljedahl, C.D.M., Crocombe, A.D., Wahab, M.A., and Ashcroft, I.A., Int. J. Adhesion and Adhesives. 27, 505-518 (2007).

[21] Wahab, M.A., Crocombe, A.D., Beevers, A., and Ebtehaj, K., Int. J. Adhesion and Adhesives. 22, 61-73 (2002).

[22] Broughton, W.R. and Hinopoulos, G., CMMT(A) 206, National Physical Laboratory (NPL). (1999).

[23] BS EN ISO 527-2:1996, British Standards Institution. (1996).

[24] Adamson, M.J., J. Mat. Sci. 15, 1736-1745 (1980).

[25] El-Sa'ad, L., Darby, M.I., and Yates, B., J. Mat. Sci. 24, 1653-1659 (1989).

[26] Kutz, M., ed. Handbook of Materials Selection. (John Wiley \& Sons, Inc., New York, 2002), Vol.

[27] BS ISO 4587:2003, British Standards Institution. (2003).

[28] Critchlow, G.W., Ashcroft, I.A., Cartwright, T., and Bahrani, D., Anodising aluminium alloy, Patent No 2421959 A, 2006, United Kingdom.

[29] Mubashar, A., Ashcroft, I.A., Critchlow, G.W., and Crocombe, A.D., International Journal of Adhesion and Adhesives. 29, 751-760 (2009).

[30] Abaqus User Subroutines Reference Manual, (Dassault Systemes Simulia Corp. Providence, RI, USA, 2008).

[31] Abaqus Analysis User's Manual, (Dassault Systemes Simulia Corp. Providence, RI, USA, 2008).

[32] Wang, C.H. and Chalkley, P., International Journal of Adhesion and Adhesives. 20, 155-164 (2000).

[33] Cardarelli, F., Materials Handbook - A Concise Desktop Reference, (Springer-Verlag London Limited, London, 2008). 2nd ed. 


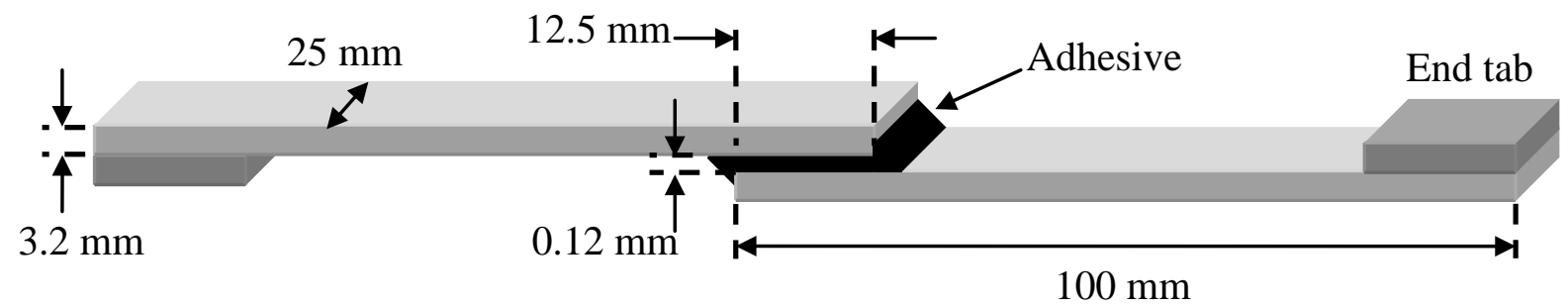

Figure 1: Single lap joint configuration and geometry (not to scale).

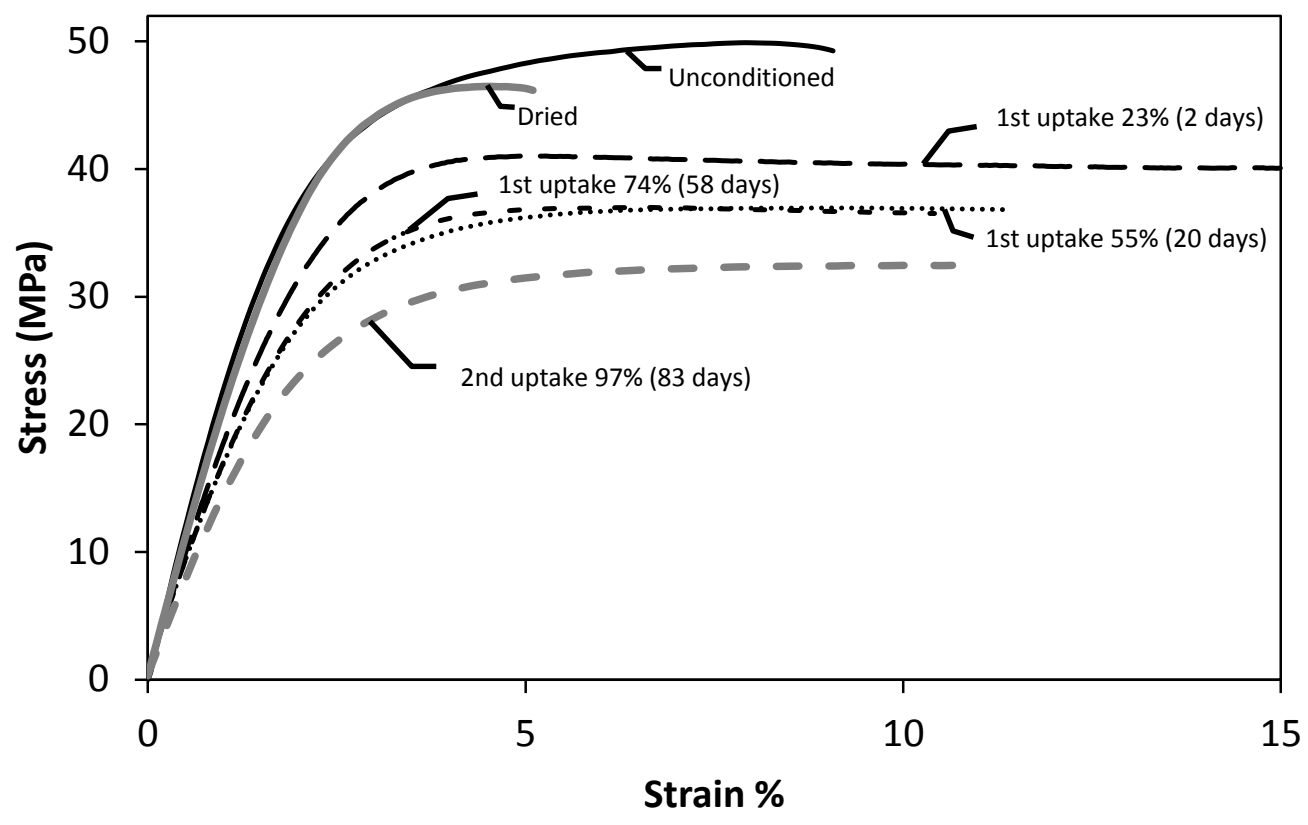

Figure 2: Moisture dependant stress vs. strain curves for the bulk adhesive at different values of $M_{t} / M_{\infty}$. 


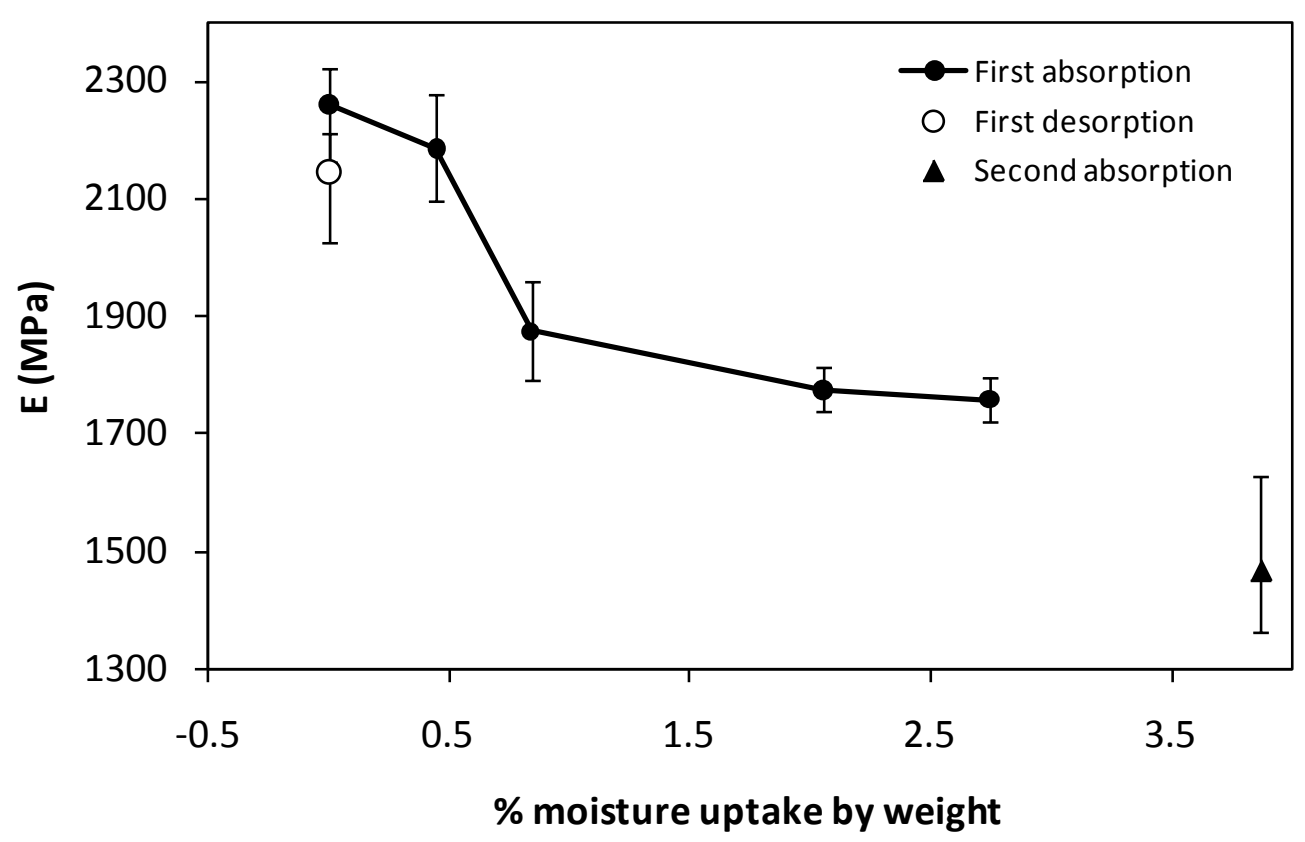

(a)

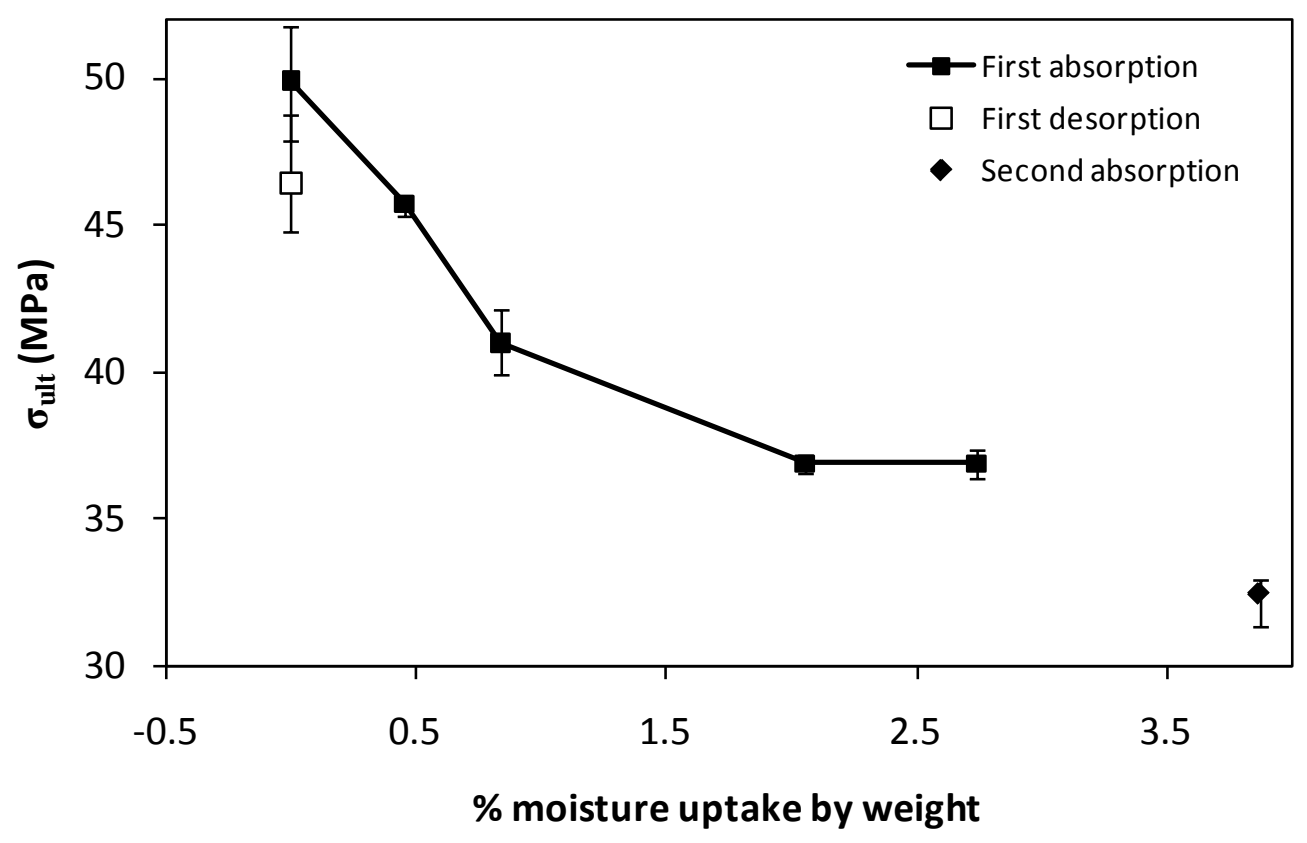

(b)

Figure 3: Effect of cyclic moisture diffusion on (a) elastic modulus and (b) ultimate tensile strength of the bulk adhesive. 


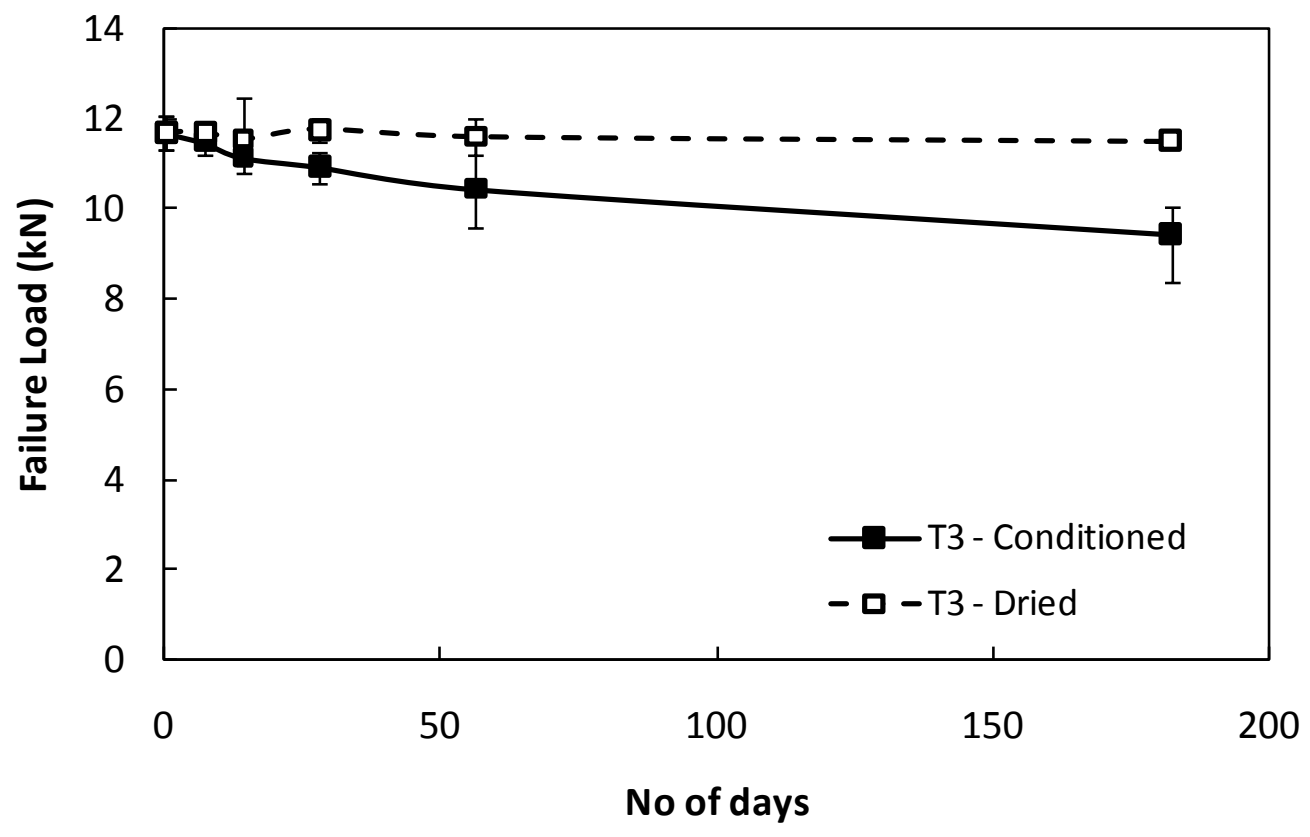

Figure 4: Failure load of single lap joints after conditioning for various time intervals at $50^{\circ} \mathrm{C}$, immersed in water.

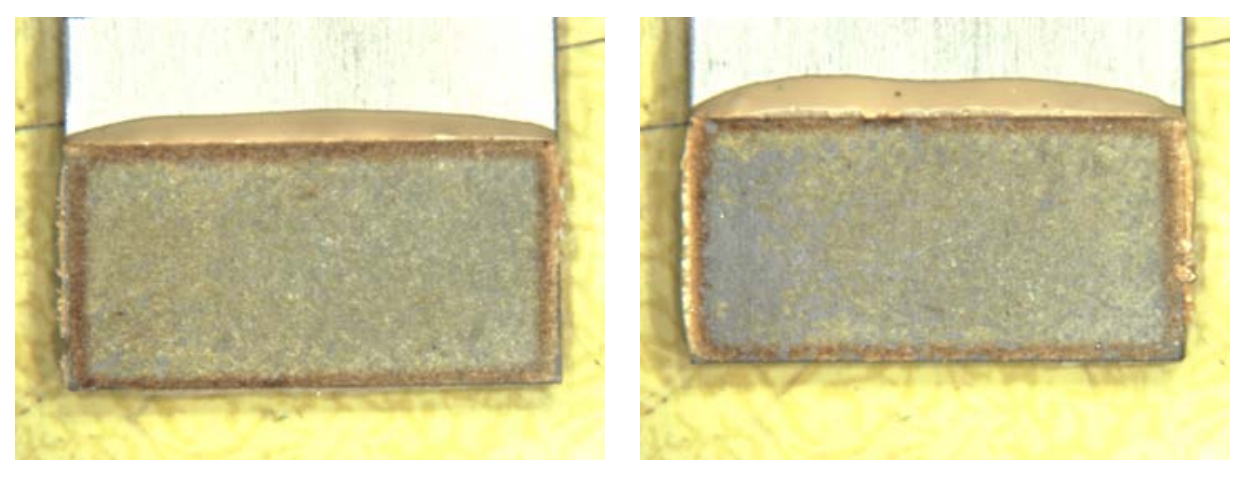

Figure 5: Failure surfaces of a single lap joint conditioned for 182 days in water. 


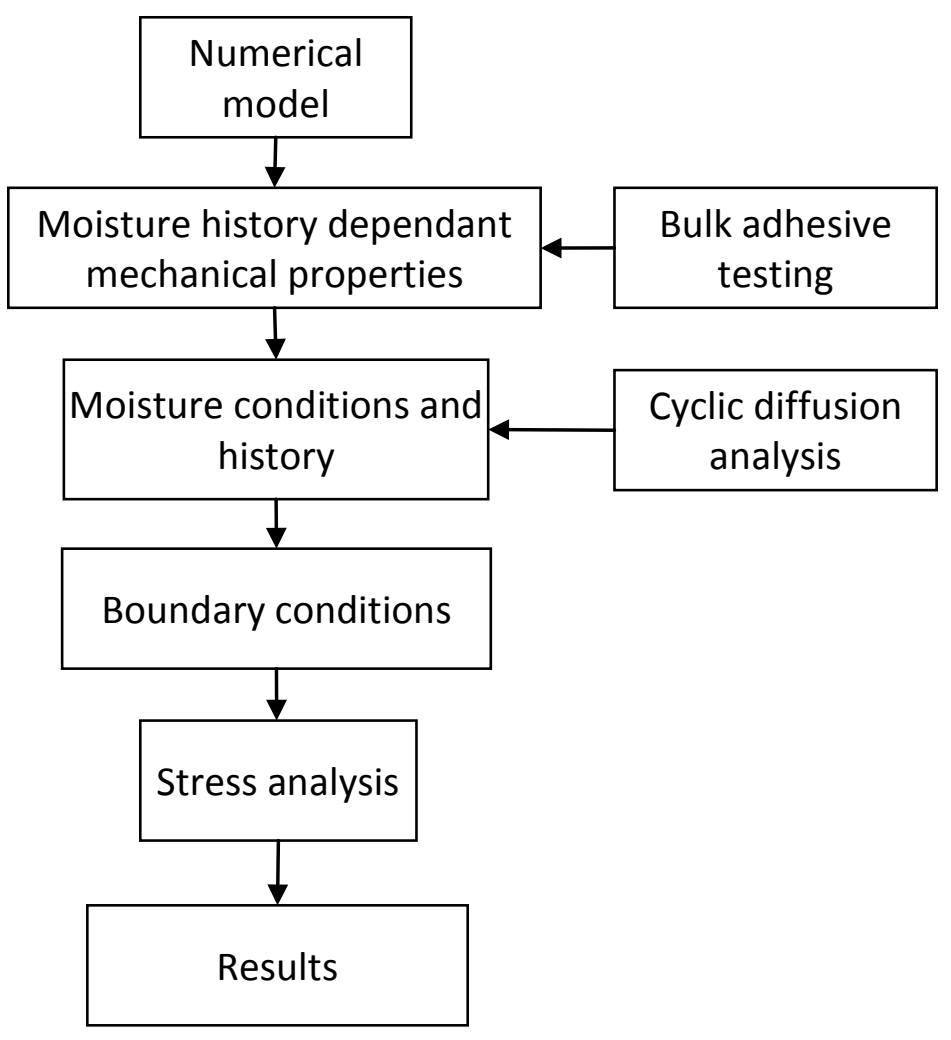

Figure 6: Methodology for implementation of cyclic stress prediction. 


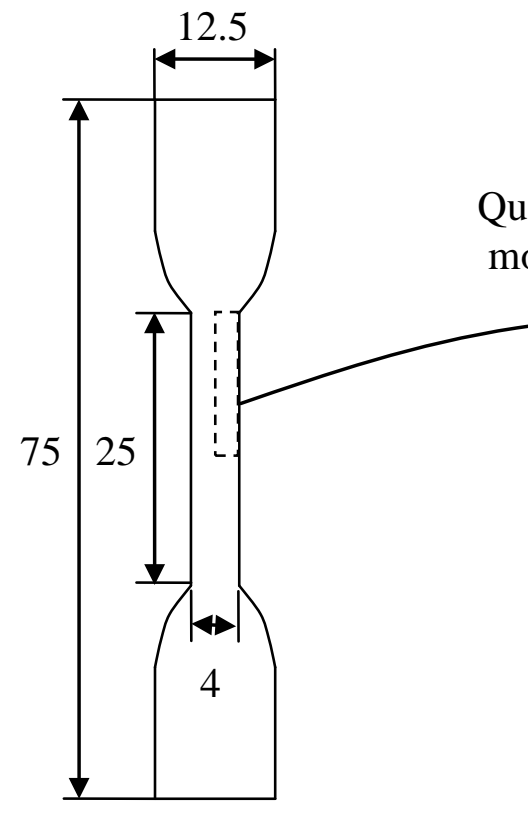

(a)

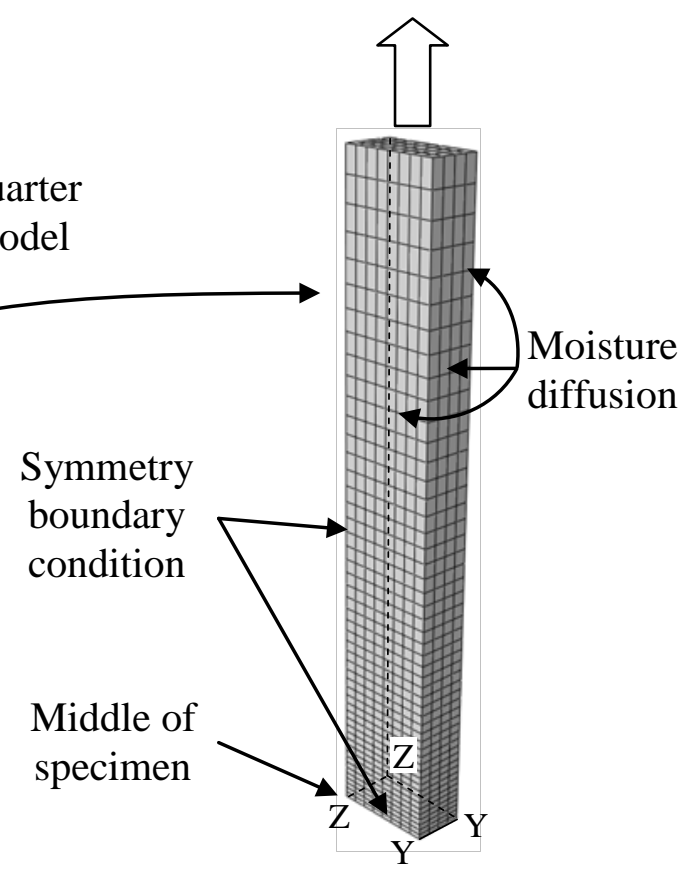

(b)

Figure 7: Tensile test specimen of the bulk adhesive (a) sample geometry (b) finite element model with boundary conditions. Dimensions are in $\mathbf{m m}$.

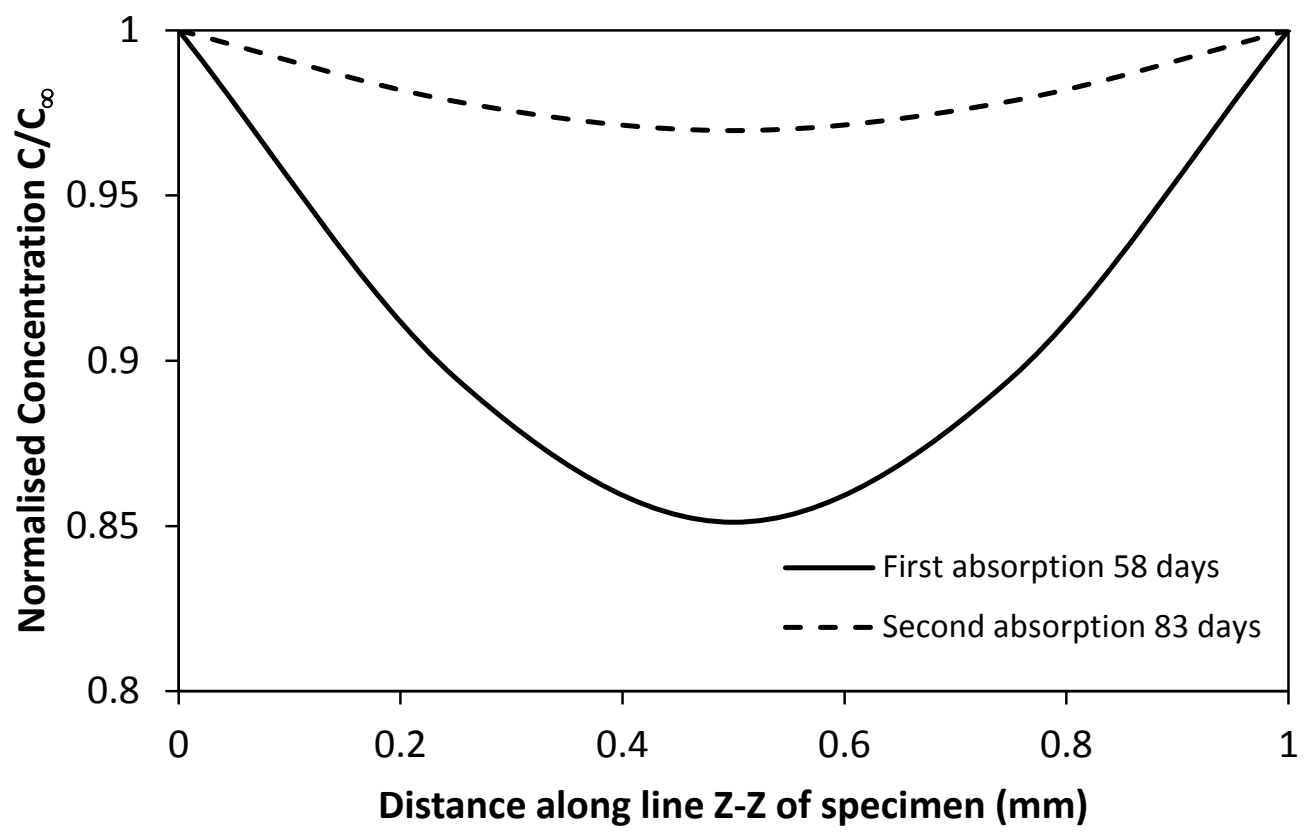

Figure 8: Normalised moisture concentration profiles in the tensile test specimen of the bulk adhesive. 


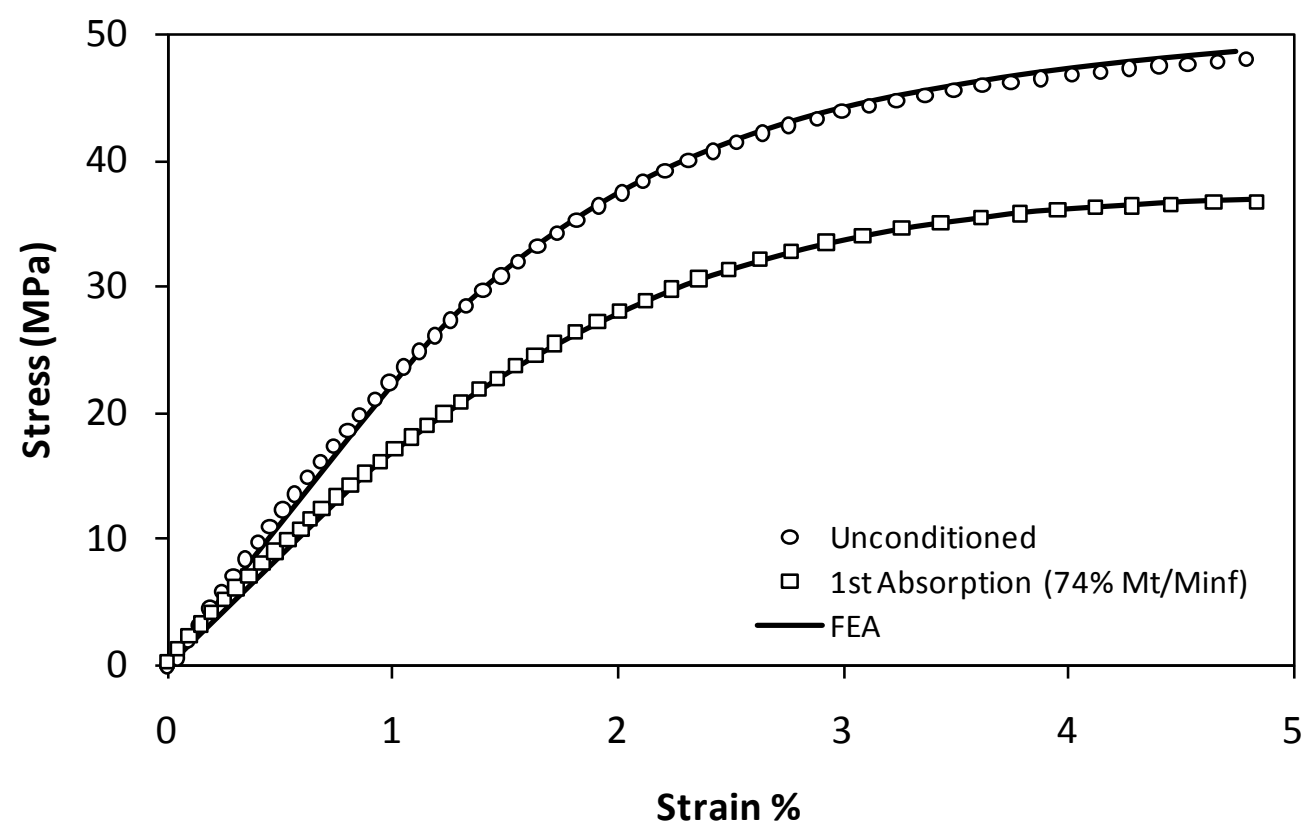

(a)

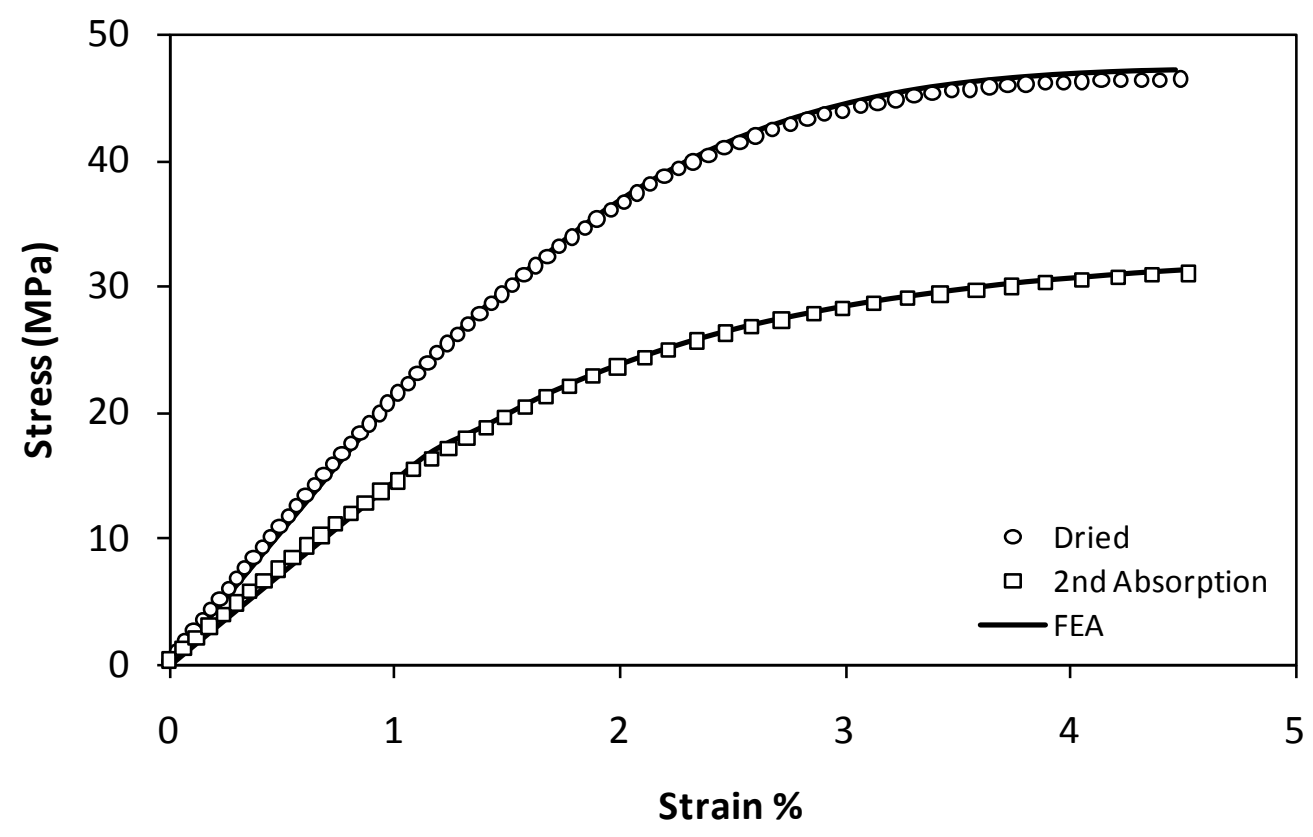

(b)

Figure 9: Comparison of experimental and finite element analysis based stress-strain curves under cyclic moisture diffusion (a) unconditioned and first absorption (b) dried and second absorption in the bulk adhesive. 


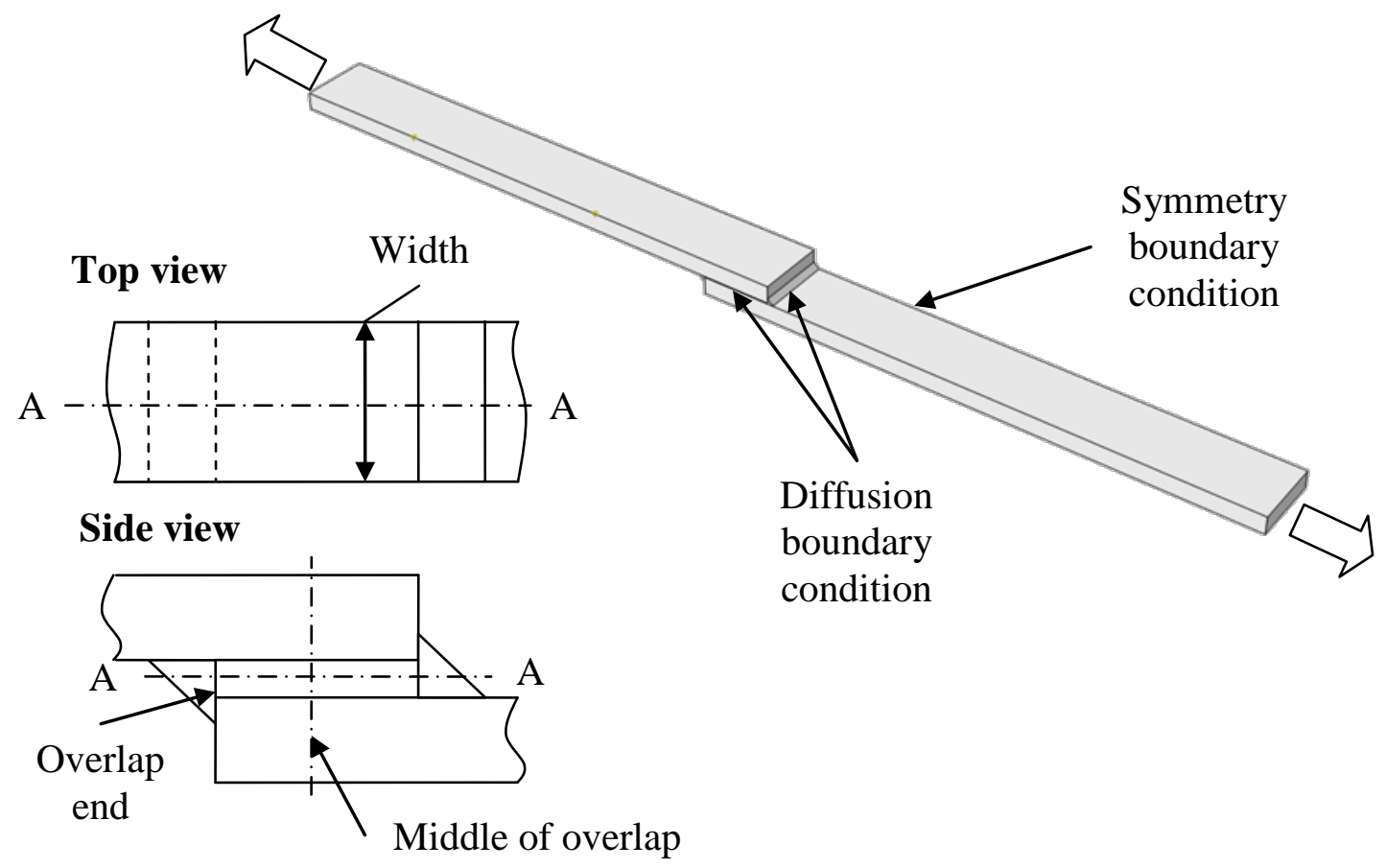

(a)

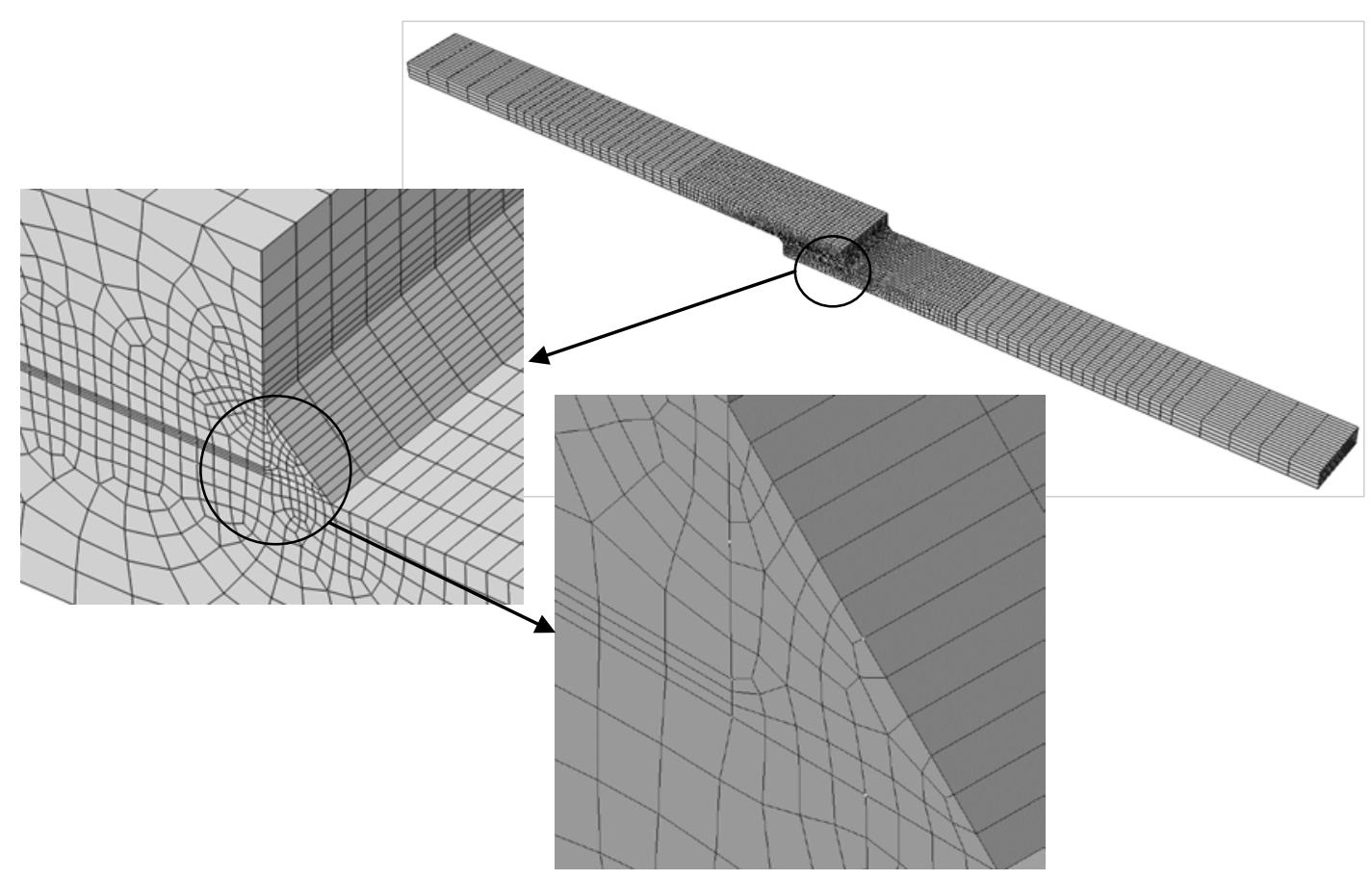

(b)

Figure 10: Finite element model of the single lap joint (a) Half model with boundary conditions (b) mesh with meshing details in the adhesive layer and fillet. 

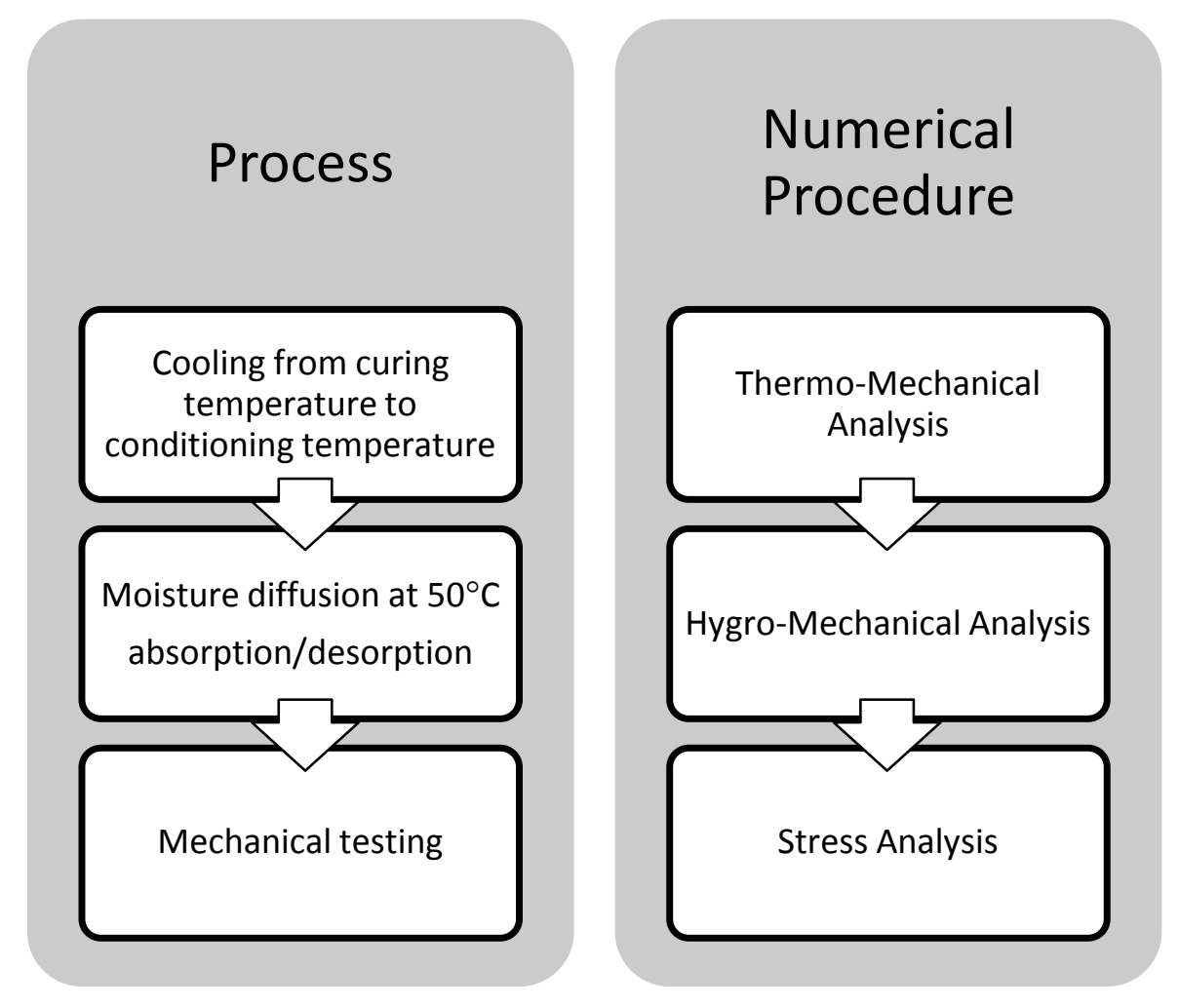

Figure 11: Process history of the single lap joint and corresponding numerical analysis procedure. 


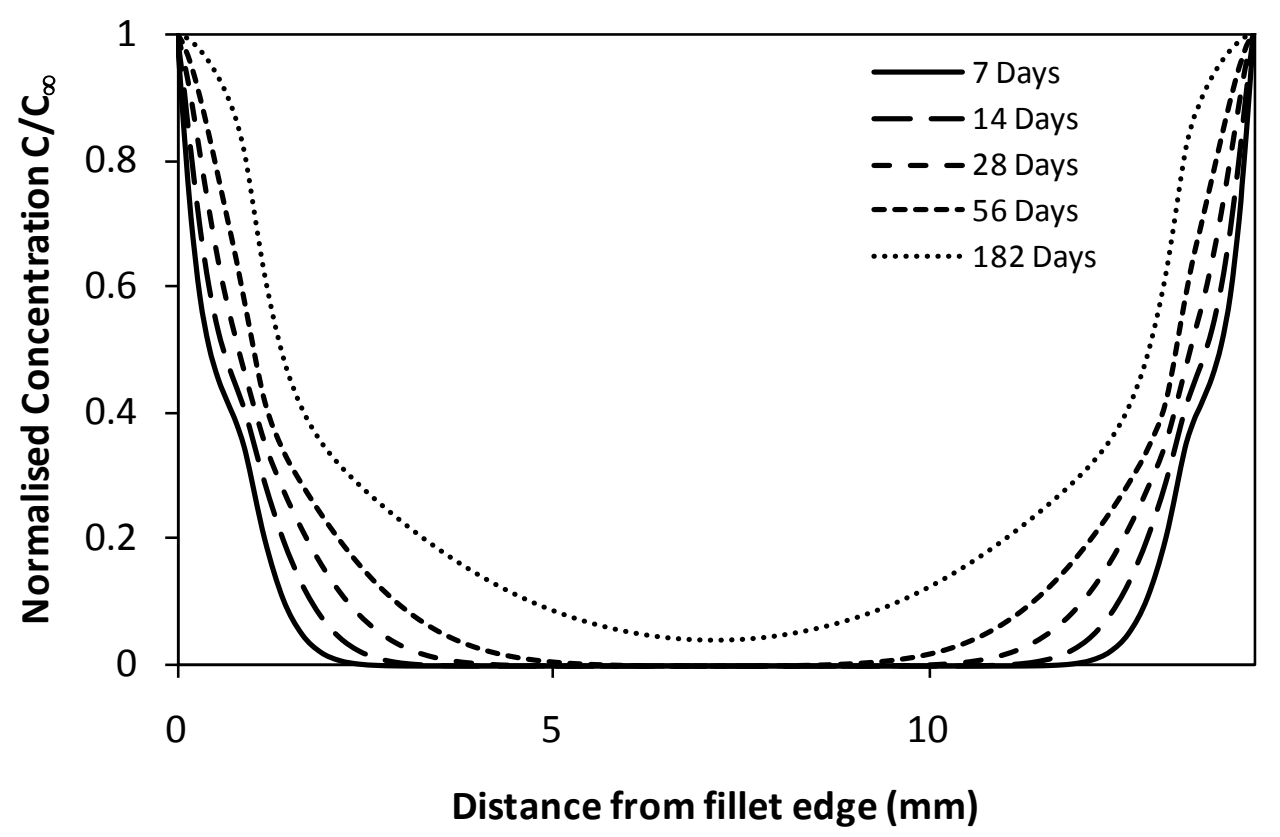

Figure 12: Normalised moisture concentration in the middle of the adhesive layer, at the centre of the joint, when conditioned at $50^{\circ} \mathrm{C}$, immersed in water.

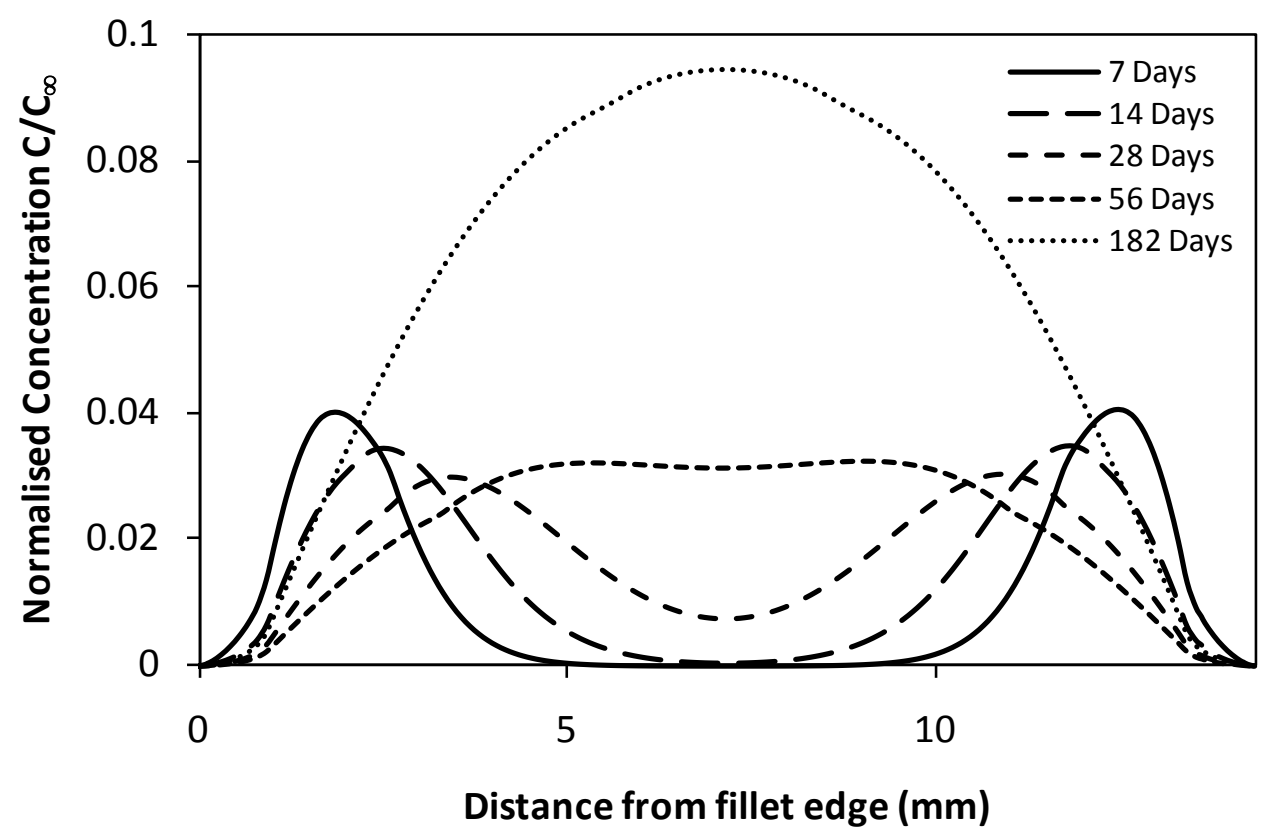

Figure 13: Normalised moisture concentration in the middle of the adhesive layer, at the centre of the joint, when conditioned at $50^{\circ} \mathrm{C}$, immersed in water and dried. 


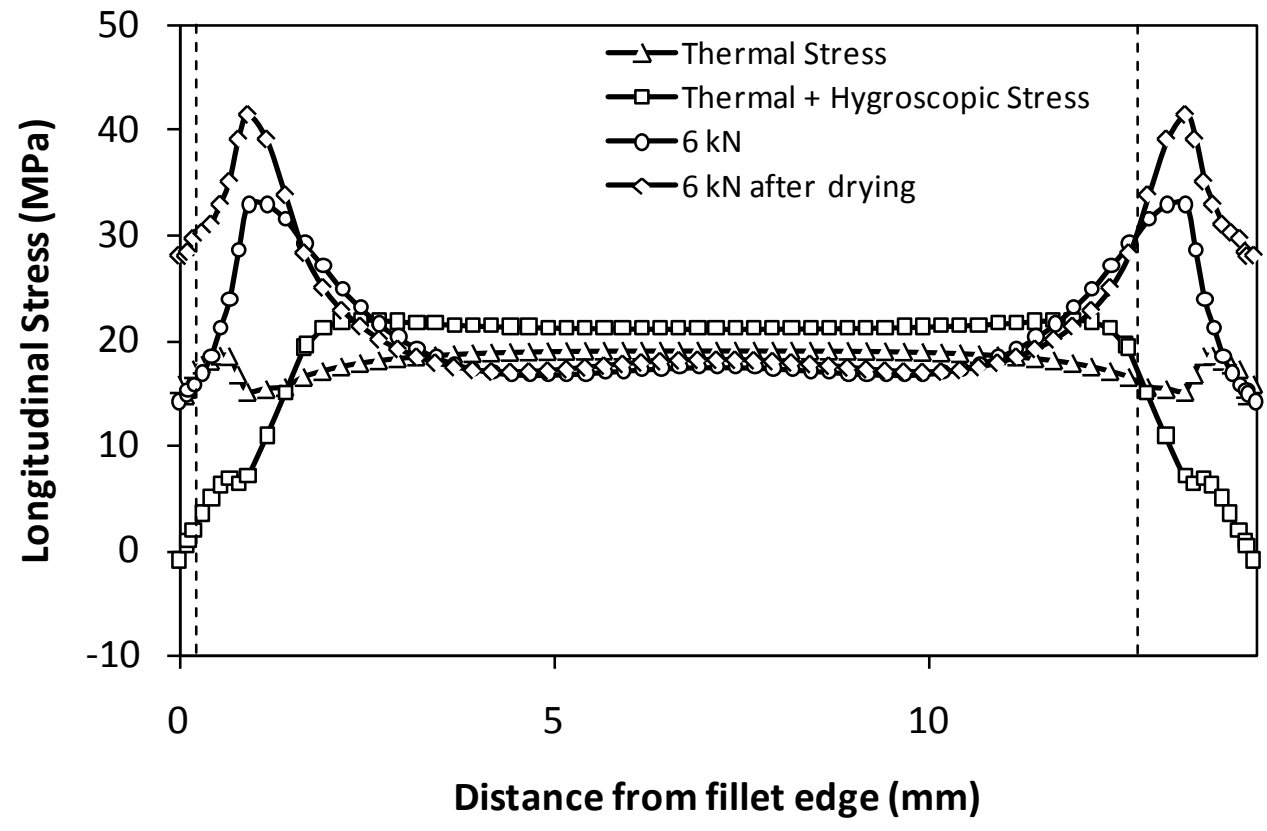

(a)

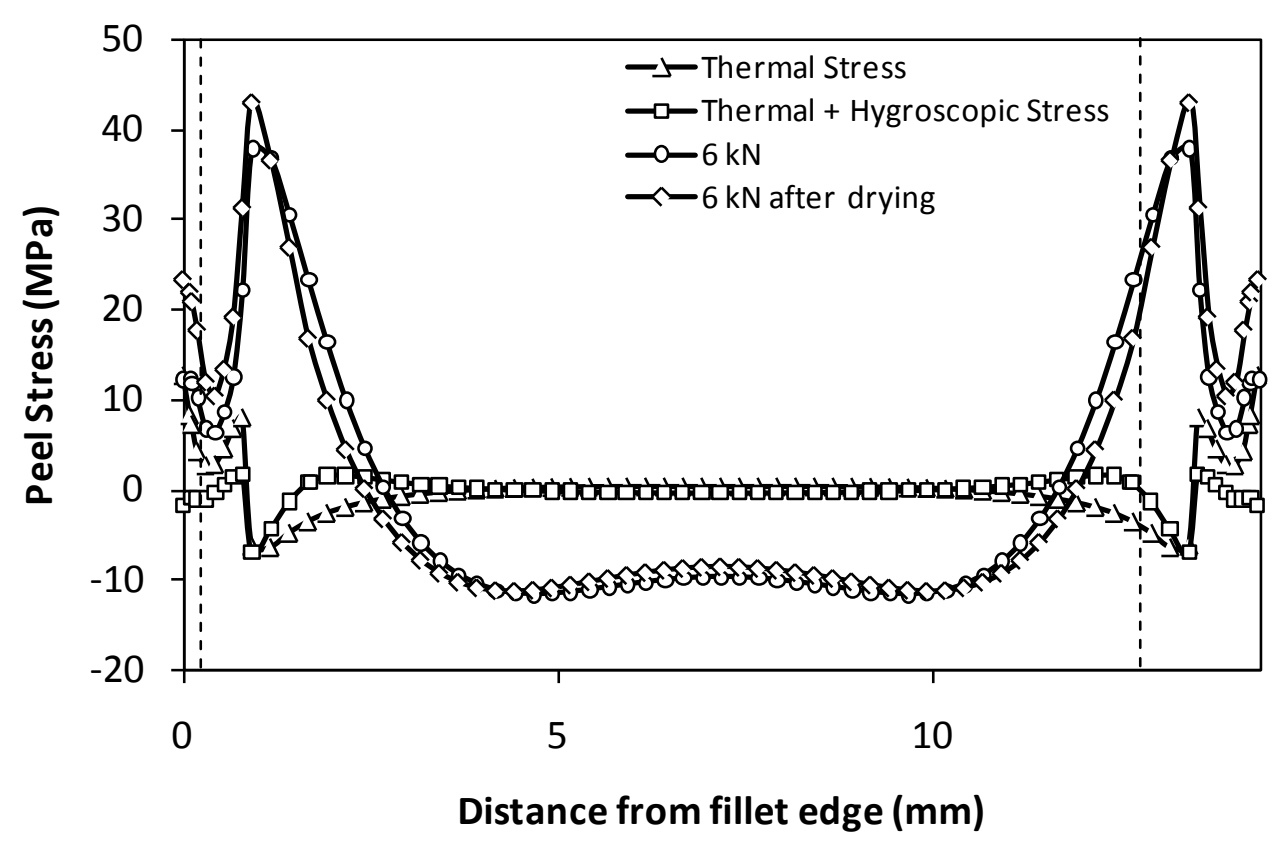

(b)

Figure 14: Development of stresses in single lap joint conditioned for 7 days at $50^{\circ} \mathrm{C}$ in water. 


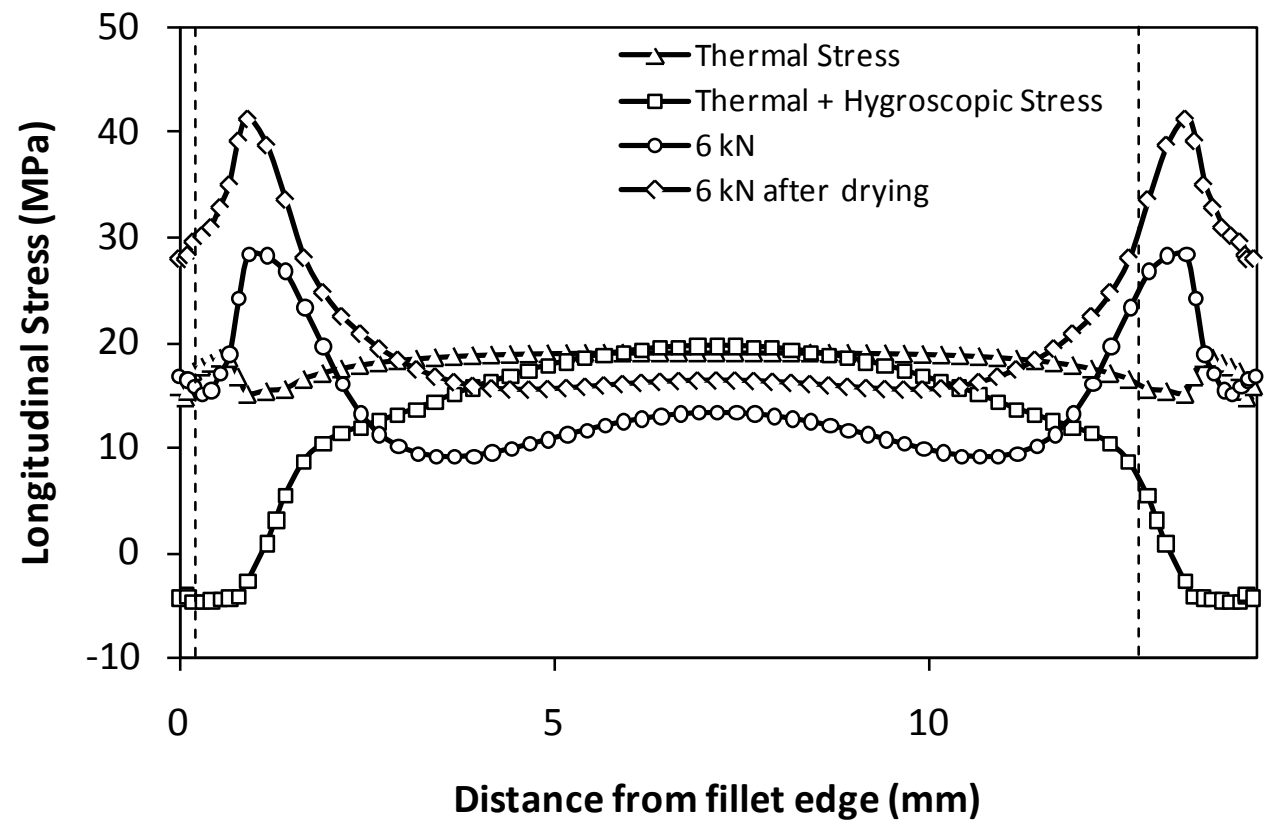

(a)

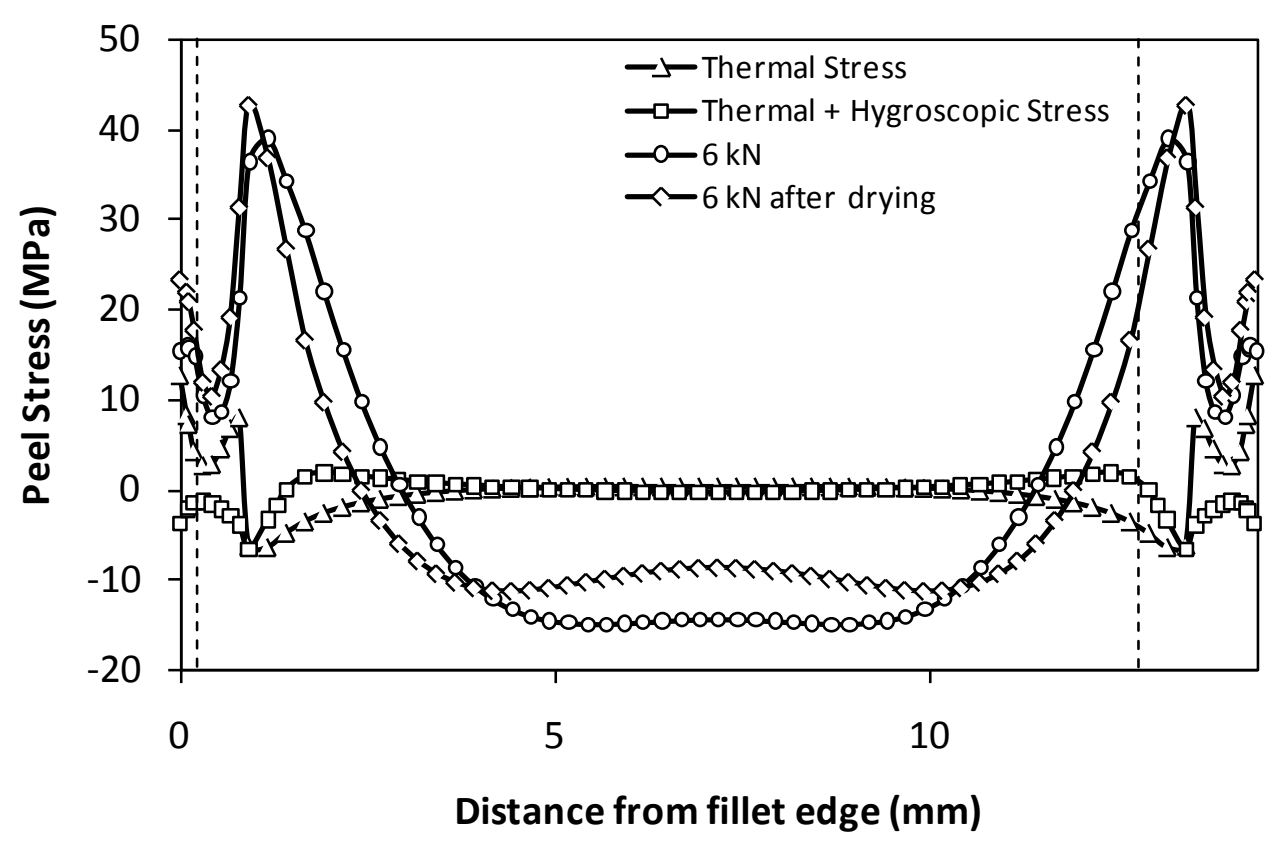

(b)

Figure 15: Development of stresses in single lap joint conditioned for 182 days at $50^{\circ} \mathrm{C}$ in water. 


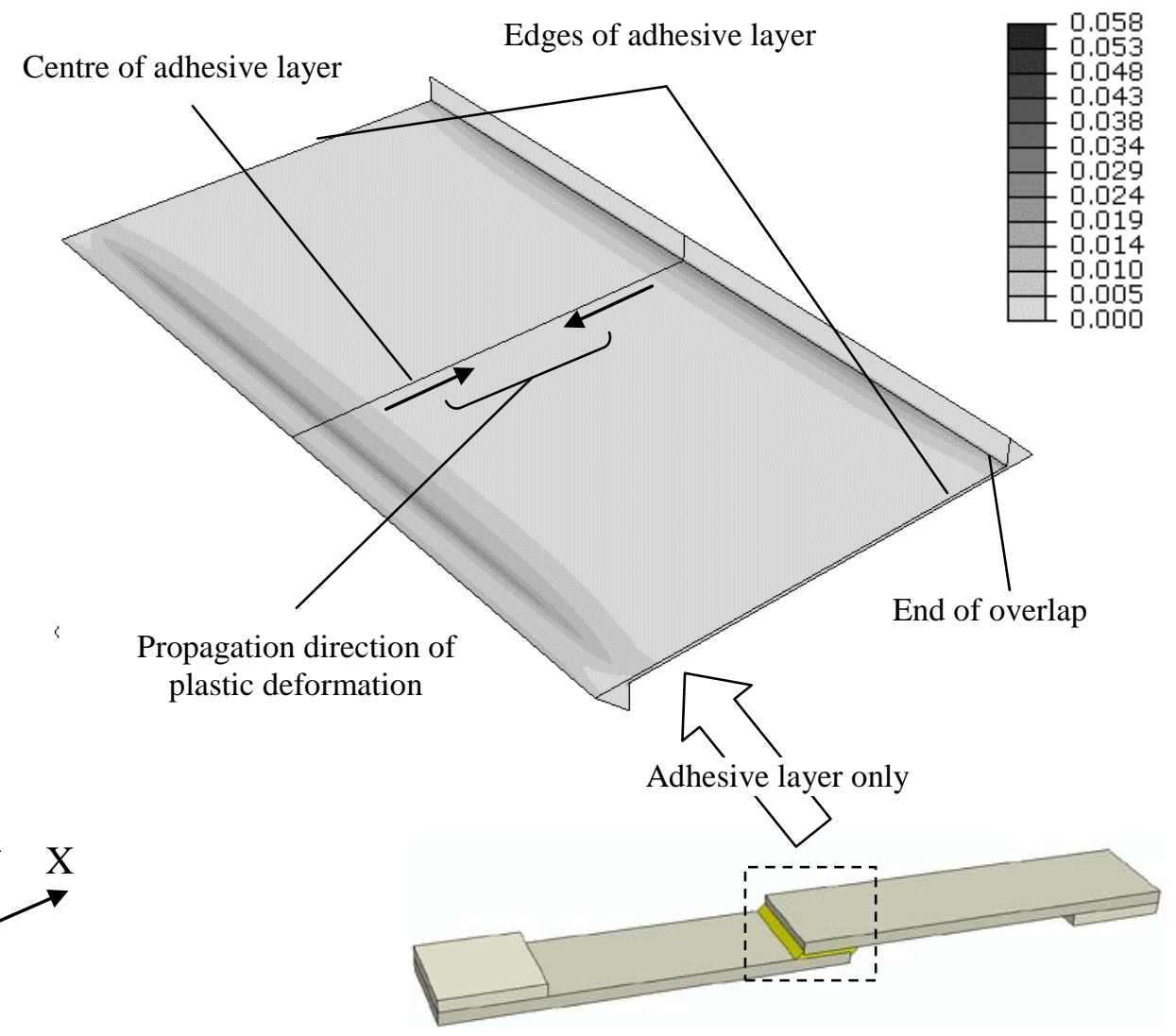

Figure 16: Equivalent plastic strain distribution in the adhesive layer of unconditioned joints after application of $6 \mathrm{kN}$ of load. 
Table 1: Mechanical and thermal properties of aluminium 2024 T3 [26].

\begin{tabular}{|l|c|}
\hline Elastic modulus (GPa) & 73 \\
\hline Poisson's ratio & 0.33 \\
\hline Yield strength (MPa) & 345 \\
\hline Coefficient of thermal expansion [33] & $23.2 \times 10^{-6} \mathrm{~K}^{-1}$ \\
\hline
\end{tabular}

Table 2: Coefficients of expansion of adhesive FM73 [5].

\begin{tabular}{|l|l|}
\hline Coefficient of thermal expansion & $7.7 \times 10^{-5} \mathrm{~K}^{-1}$ \\
\hline Coefficient of hygroscopic expansion & $0.0021\left(\%^{-1}\right)$ \\
\hline
\end{tabular}

